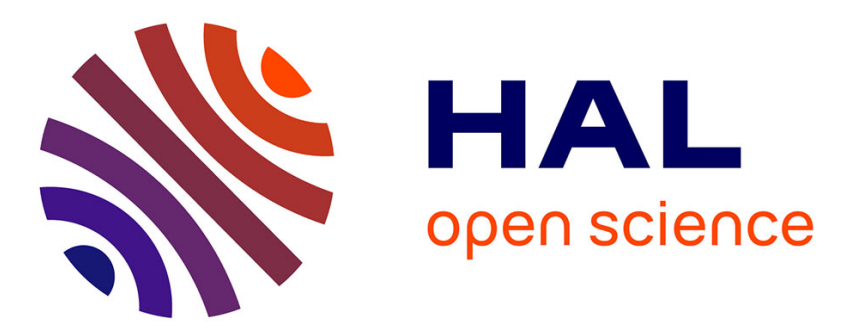

\title{
Increasing the scope and precision of the steady-state photocarrier grating technique by measuring the photocurrents at several voltages
}

Leonardo Kopprio, Christophe Longeaud

\section{- To cite this version:}

Leonardo Kopprio, Christophe Longeaud. Increasing the scope and precision of the steady-state photocarrier grating technique by measuring the photocurrents at several voltages. Semiconductor Science and Technology, 2021, 36 (12), pp.125003. 10.1088/1361-6641/ac271b . hal-03404160

\author{
HAL Id: hal-03404160 \\ https://hal-centralesupelec.archives-ouvertes.fr/hal-03404160
}

Submitted on 26 Oct 2021

HAL is a multi-disciplinary open access archive for the deposit and dissemination of scientific research documents, whether they are published or not. The documents may come from teaching and research institutions in France or abroad, or from public or private research centers.
L'archive ouverte pluridisciplinaire HAL, est destinée au dépôt et à la diffusion de documents scientifiques de niveau recherche, publiés ou non, émanant des établissements d'enseignement et de recherche français ou étrangers, des laboratoires publics ou privés. 


\title{
Increasing the scope and precision of the steady-state photocarrier grating technique by measuring the photocurrents at several voltages
}

\author{
Leonardo Kopprio ${ }^{1, *}$ and Christophe Longeaud ${ }^{2}$ \\ 1 Institut Photovoltaïque d'Île-de-France (IPVF), 18 Bd Thomas Gobert, 91120 Palaiseau, France. \\ ${ }^{2}$ Group of Electrical Engineering - Paris (GeePs), CNRS, CentraleSupelec, Université Paris-Saclay, Sorbonne Université, \\ 11 rue Joliot Curie, 91190 Gif sur Yvette, France. \\ E-mail: leonardokopprio@gmail.com
}

\begin{abstract}
The steady-state photocarrier grating (SSPG) experiment is a popular technique for extracting the minority carrier diffusion length of photoconductive thin films in coplanar configuration. The diffusion length is basically obtained from the measurement of the steady-state photocurrent produced by a low applied voltage while the material is illuminated by two monochromatic laser beams of different intensities that interfere between the electrical contacts of the sample. Despite its simplicity and popularity, it is well known that the technique can overestimate the minority carrier diffusion length in some samples. In this paper, we show that the precision of the technique can be substantially increased by performing the same experiment at different voltages. Additionally, we show that the density of states at the majority carrier quasi-Fermi energy, and that the ratio between the recombination states' capture coefficient and mobility of majority carriers can be extracted by combining the SSPG and the steady-state photocurrent (SSPC) experiments. First, we show that the procedures found in the literature for correcting the overestimation produced by the standard technique do not work properly due to an oversimplification in the modeling. Then, we use a numerical simulation of an unintentionallydoped hydrogenated-amorphous-silicon-like material to evaluate the precision of the new formulas and procedures presented. We clarify the conditions under which the standard SSPG technique produces large overestimations. In these cases, we show that the precision of the new procedure can be more than ten times higher. Finally, we use the standard and the new method to characterize a hydrogenated amorphous (a-Si:H) and a hydrogenated polymorphous (pmSi:H) silicon samples at different temperatures. We observe that the overestimations produced by the standard technique increases with the ratio between the majority and minority carriers diffusion lengths and the ratio between the recombination states' capture coefficient and mobility of majority carriers.
\end{abstract}

Keywords: transport properties, diffusion length, characterization, photoconductors, thin films. 


\section{Introduction}

The excess-carrier properties of a photoexcited semiconductor are important indicators of its quality with respect to applications in optoelectronic devices such as solar cells. In contrast to the majority-carrier properties, which can be determined rather straightforwardly by the steady-state photocurrent (SSPC) experiment, the minority-carrier properties can only be revealed by more sophisticated techniques, such as the steady-state photocarrier grating (SSPG). In this case, the stationary photocurrent is measured while the semiconductor is illuminated by two coherent light beams of different intensities, which interfere inside the material to form a light grating in a uniform background of higher intensity.

The SSPG technique was initially proposed by Ritter, Zeldov and Weiser [1] for the estimation of the ambipolar diffusion length $\left(L_{a m b}\right)$ of photoconductive materials in coplanar configuration, i.e., with two electrical contacts deposited on the same surface of the film. In an extrinsic semiconductor, the minority-carrier diffusion length is proportional to the ambipolar diffusion length $\left(\sim \sqrt{2} L_{a m b}\right)$ and can be easily estimated from its value.

In order to extract $L_{a m b}$ for a fixed temperature and uniform photon flux using SSPG, it is necessary to measure the decreases in the SSPC produced by light gratings with different periods. The larger the period of the light grating $(\Lambda)$, the higher is the decrease in the photocurrent perpendicular to the grating fringes. $L_{a m b}$ is obtained by fitting the stationary decreases of the photocurrent versus the $\Lambda$ values with a theoretical equation known as the Ritter, Zeldov, and Weiser (RZW) equation. This is the initial approach of Ritter et al. [1], which is only valid when the applied voltage is low enough and the material is in the "lifetime regime", i.e., when the dielectric relaxation time is much shorter than the carrier lifetimes (we call this approach the low-voltage SSPG). In this case, the transport of photocarriers is ambipolar, i.e., the diffusion of electrons and holes is correlated due to their mutual attraction.

For some samples under quite usual experimental conditions, the material is not in the "lifetime regime" anymore, and the length obtained from the low-voltage SSPG overestimates the true ambipolar diffusion length of the photocarriers. In this case, it is said that the material is in the "relaxation regime", the transport is bipolar instead of ambipolar, and there is a higher space-charge distribution inside the material [2-4]. To correct the overestimation of $L_{a m b}$, Ritter et al. [2] proposed measuring the SSPC and the small-signal decay of the photocurrent (SSDP). A similar way to correct the overestimation was suggested by Hattori et al. [4], in which SSPC is also measured, but the frequency-resolved photocurrent is used instead of the SSDP experiment.

All the previously cited publications on SSPG analyze the technique from a phenomenological point of view, which does not distinguish between trapped and free photocarriers. Later analyses, such as the one from Abel et al. [5] or Schmidt and Longeaud [6], are based on the multiple trapping (MT) model, which explicitly separates free from trapped carriers. This model assumes that the carrier transport occurs in extended-states located outside the mobility gap, while the localized-states within the mobility gap act exclusively as traps and recombination centers for the free carriers. At present, there is a consensus on the validity of the MT model in high-quality hydrogenated-amorphous-silicon-like materials for sufficiently high temperatures and generation rates, such as the ones used in this manuscript [5,7,8]. Balberg [9] 
and Shah et al. [10] have started investigating the link between these two formalisms applied to SSPG, but to our knowledge, a more detailed comparison is still missing in the bibliography.

We start by comparing the equations obtained from each of the two mentioned formalisms. We find out that they are not equivalent. One of the assumptions made in the phenomenological approach is not verified within the MT model. Because of this wrong assumption, the procedures previously proposed to correct the overestimation of $L_{a m b}$ produced by the low-voltage SSPG $[2,4]$, do not work properly. In this manuscript, we propose a new procedure for obtaining good estimations of the minority-carrier diffusion length, the ratio between the recombination states capture coefficient and mobility of the majority carriers, and the density of states at the majority carrier quasi-Fermi energy. In order to determine these parameters, it is necessary to measure SSPC, and the stationary decreases in the photocurrent generated by different grating periods under different applied voltages (we call this new approach the multi-voltage SSPG).

Over the years, the SSPG technique has become a standard method for material characterization in several laboratories [11], where it has been effectively applied to a-Si:H and its alloys, hydrogenated microcrystalline silicon and germanium, chalcopyrite and III-V semiconductors [12,13], and also to hybrid perovskites [14-16]. We focus the analysis on a-Si:Hlike materials because are one of the most thoroughly studied thin-film materials in the bibliography, and there is a broad consensus on their basic optoelectronic modeling [17-19]. The results of this manuscript may be easily generalized to other extrinsic semiconductors, especially to those where the standard SSPG has already been applied.

The content and organization of this work are detailed as follows. Section 2 provides a theoretical analysis of the SSPG technique. First, we present the expression of the current density generated by the SSPG illumination in a photoconductive material under an arbitrary applied electric field (2.1). Then, we describe the experimental setup necessary for the correct implementation of the technique (2.2). In subsection 2.3 we deduce the RZW equation from the general equation and detail the problem of the phenomenological model used in previous papers. In subsection 2.4, we simplify the general equations assuming a non-intrinsic material, which allows us to deduce, in subsection 2.5 , an equation for the diffusion length of the minority carriers. The equation is a function of parameters that can be obtained from the multi-voltage SSPG, and in subsection 2.6, we describe the best way of extracting these parameters. In subsection 2.7, we deduce a formula to obtain the ratio between the majority-carrier capture coefficient of the recombination states and the majority-carrier mobility. In subsection 2.8, we present a new quantitative definition of the "lifetime regime".

Section 3 mainly evaluates the precision of the methods and formulas presented in the previous section using a numerical simulation of an unintentionally-doped a-Si:H-like material. Subsection 3.1 describes the details of the numerical simulation. First, we evaluate the precision of the standard low-voltage approach (3.2), and then we evaluate the new method and formulas presented in this paper (3.3). We observe that the multi-voltage method is much more precise than the low-voltage approach, especially at low temperatures. The new method allows obtaining the minority carrier diffusion length with errors lower than $5 \%$. On the other hand, the formula for the ratio between the recombination states' capture coefficient and mobility of majority carriers presents only an excellent precision in the low-temperature range, where the ratio between majority and minority carrier concentration is high. 
The experimental results are presented in section 4, where the low and multi-voltage procedures are applied to a hydrogenated amorphous (a-Si:H) and a hydrogenated polymorphous (pm-Si:H) silicon samples. Subsection 4.1 describes the experimental details and evaluates the Ohmicity of the electrical contacts, a necessary condition for applying the multivoltage approach. An evaluation of the experimental data's fitting with the new formulas is presented in subsection 4.2. In subsection 4.3, we present the final results for each sample and compare their characteristics. The experimental results are consistent with those observed in the numerical simulation. The overestimation of the minority carrier diffusion length produced by the low-voltage approach is larger in the pm-Si:H sample due to its higher ratio between the recombination states' capture coefficient and mobility of majority carriers.

The conclusion of this work is presented in section 5 , where we summarize the main results of this paper. The auxiliary mathematical probes and deductions are given in three appendixes to facilitate the reading of the manuscript.

\section{Theoretical development}

\subsection{General equation}

In the SSPG technique, a photoconductor is illuminated between two Ohmic coplanar electrical contacts with a monochromatic light, composed of a sinusoidal grating parallel to the contacts and a uniform background of much higher intensity. When the energy of the photons is higher than the band-gap of the semiconductor, the steady-state generation rate of photocarriers inside the material is proportional to the light intensity [20]. As a consequence, the steady-state generation rate, averaged in the incident light direction, is given by

$$
G=G_{0}+\Delta G_{0} \cos (k x)
$$

where $G_{0} \gg \Delta G_{0}, x$ is the spatial coordinate taken perpendicularly to the electrodes and $k$ is related to the period of the light grating $\Lambda$, by $k=2 \pi / \Lambda$.

Using the MT model, Schmidt and Longeaud [6] obtained the first-order expression of the steady-state current density $J$, induced in the material under the SSPG illumination while applying an external potential difference between the contacts. The higher-order terms of the current density can be neglected because $G_{0} \gg \Delta G_{0}$ [5]. In this case, $J$ is composed of two terms,

$$
J=J_{0}+\Delta J
$$

The first one is only due to the uniform illumination and is given by $J_{0}=\sigma_{0} \xi_{\text {ext }}$, where $\xi_{\text {ext }}$ is the externally applied electric field, and $\sigma_{0}$ is the electrical conductivity of the material under the generation rate $G_{0}$,

$$
\sigma_{0}=q\left(\mu_{n} n_{0}+\mu_{p} p_{0}\right) .
$$

$q$ is the absolute value of the electron charge, $n_{0}$ and $p_{0}$ are the steady-state uniform freeelectrons and free-holes concentrations in the material, respectively, and $\mu_{n}$ and $\mu_{p}$ are their corresponding mobilities. 
The grating produces a reduction of the current induced by the uniform illumination, as can be appreciated in the equation for $\Delta J$,

$$
\Delta J=\frac{-q^{2} \Delta G_{0}^{2} J_{0}}{2 \sigma_{0}^{2}\left(\omega_{a}^{2}+\omega_{b}^{2}\right)}\left[\left(\mu_{n}\left(1+Q^{+}\right)+\mu_{p}\left(1+Q^{-}\right)\right)+2 k^{2} \frac{\varepsilon k_{b} T}{q \sigma_{0}} \mu_{n} \mu_{p}\right]\left(\mu_{n}\left(1+Q^{+}\right)+\mu_{p}\left(1+Q^{-}\right)\right),
$$

which has the opposite sign to $J_{0} . \varepsilon$ is the permittivity of the material, $k_{b}$ is the Boltzmann constant, $T$ is the absolute temperature, and the frequencies $\omega_{a}$ and $\omega_{b}$, are given by

$$
\begin{gathered}
\omega_{a}=\left(\frac{\left(1+Q^{-}\right)}{\tau_{p}^{r}}+\frac{\left(1+Q^{+}\right)}{\tau_{n}^{r}}\right)+\frac{\varepsilon k^{2}}{\sigma_{0}}\left[\left(\frac{\mu_{p}}{\tau_{n}^{r}}+\frac{\mu_{n}}{\tau_{p}^{r}}\right) \frac{k_{b} T}{q}+\frac{k_{b} T}{q \varepsilon} q \mu_{p} \mu_{n}\left(p_{0}\left(1+Q^{+}\right)+n_{0}\left(1+Q^{-}\right)\right)+\right. \\
\left.\mu_{n} \mu_{p} \xi_{\text {ext }}^{2}+k^{2} \mu_{n} \mu_{p}\left(\frac{k_{b} T}{q}\right)^{2}\right] \\
\omega_{b}=\frac{\varepsilon k \xi_{\text {ext }}}{\sigma_{0}}\left[\frac{\mu_{p}}{\tau_{n}^{r}}-\frac{\mu_{n}}{\tau_{p}^{r}}+\frac{q \mu_{n} \mu_{p}}{\varepsilon}\left(n_{0}\left(1+Q^{-}\right)-p_{0}\left(1+Q^{+}\right)\right)\right]
\end{gathered}
$$

The positive dimensionless parameters $Q^{+}$and $Q^{-}$correspond to the derivative of the total trapped-charge with respect to the positive and negative free-charge, respectively,

$$
Q^{+}=\left[\frac{q \partial\left(p^{t}-n^{t}\right)}{q \partial p}\right]_{G_{0}}, \quad Q^{-}=\left[\frac{q \partial\left(p^{t}-n^{t}\right)}{-q \partial n}\right]_{G_{0}} .
$$

$n^{t}\left(p^{t}\right)$ is the concentration of trapped-electrons (trapped-holes) and $n(p)$ is the concentration of free-electrons (free-holes) in the material. The frequencies $1 / \tau_{n}^{r}$ and $1 / \tau_{p}^{r}$ correspond to the derivatives of the recombination rate $r$, with respect to the free-electron and free-hole concentrations, respectively,

$$
1 / \tau_{n}^{r}=\left[\frac{\partial r}{\partial n}\right]_{G_{0}}, \quad 1 / \tau_{p}^{r}=\left[\frac{\partial r}{\partial p}\right]_{G_{0}}
$$

Although the nomenclature is different, equations (4-6) are equivalent to equations (8-10) of Ref. [6].

The logarithmic derivatives of the free-carrier concentrations with respect to the uniform generation rate,

$$
\gamma_{n}=\partial\left[\ln \left(n_{0}\right)\right] / \partial\left[\ln \left(G_{0}\right)\right], \quad \gamma_{p}=\partial\left[\ln \left(p_{0}\right)\right] / \partial\left[\ln \left(G_{0}\right)\right],
$$

are related to the parameters $Q^{+}$and $Q^{-}$, in accordance with the differential neutrality equation,

$$
\left(1+Q^{+}\right) \gamma_{p} p_{0}=\left(1+Q^{-}\right) \gamma_{n} n_{0}
$$

obtained by producing a differential variation of $G_{0}$. Similarly, $\gamma_{n}$ and $\gamma_{p}$ are also related to the recombination times $\tau_{n}^{r}$ and $\tau_{p}^{r}$, according to the differential continuity equation,

$$
\frac{\gamma_{p} p_{0}}{\tau_{p}^{r}}+\frac{\gamma_{n} n_{0}}{\tau_{n}^{r}}=G_{0}
$$

In Appendix A, we present the deduction of equations (10) and (11), and the expressions of the parameters $Q^{+}, Q^{-}, \tau_{n}^{r}, \tau_{p}^{r}, \gamma_{n}$ and $\gamma_{p}$ for an arbitrary density of monovalent localized states in the band-gap of the semiconductor. 
Using equations (10) and (11) and some algebra, we can rewrite equations (4)-(6) in a compact way, which makes explicit the dependence of $\Delta J / J_{0}$ with $k^{2}$ and $\xi_{\text {ext }}^{2}$,

$$
\frac{\Delta J}{J_{0}}=\frac{-\theta_{0}^{2}\left[1+L_{d D}^{4} k^{2} / L_{0}^{2}\right]}{\left\{1+\left[L_{D}^{2}+\frac{L_{d D}^{4}}{2}\left\{k^{2}+\left(\frac{q \xi_{e x t}}{k_{b} T}\right)^{2}\right\} k^{2}\right\}^{2}+\left(\frac{q \xi_{\text {ext }}}{k_{b} T}\right)^{2} L_{d}^{4} k^{2}\right.} .
$$

$\theta_{0}^{2}, L_{0}^{2}, L_{D}^{2}, L_{d D}^{2}$ and $L_{d}^{2}$ are given by,

$$
\begin{gathered}
\theta_{0}^{2}=\frac{\gamma_{\sigma}^{2} \Delta G_{0}^{2}}{2 G_{0}^{2}}, \\
L_{0}^{2}=\left(\frac{k_{b} T}{q}\right) \frac{\gamma_{\sigma} \sigma_{0}}{q G_{0}}, \\
L_{D}^{2}=\frac{\left(\gamma_{n}+\gamma_{p}\right)}{2} L_{a}^{2}+\frac{\tau_{d} \gamma_{n} L_{n}^{2}}{\left(1+Q^{+}\right) \tau_{p}^{r}}+\frac{\tau_{d} \gamma_{p} L_{p}^{2}}{\left(1+Q^{-}\right) \tau_{n}^{r}} \\
L_{d D}^{2}=L_{n} L_{p} \sqrt{\frac{2 \tau_{d} \gamma_{n}}{\left(1+Q^{+}\right) \tau_{p}}}=L_{n} L_{p} \sqrt{\frac{2 \tau_{d} \gamma_{p}}{\left(1+Q^{-}\right) \tau_{n}}}, \\
L_{d}^{2}=\frac{\left(\gamma_{n}-\gamma_{p}\right)}{2} L_{a}^{2}+\frac{\tau_{d} \gamma_{n} L_{n}^{2}}{\left(1+Q^{+}\right) \tau_{p}^{r}}-\frac{\tau_{d} \gamma_{p} L_{p}^{2}}{\left(1+Q^{-}\right) \tau_{n}^{r}} .
\end{gathered}
$$

$\gamma_{\sigma}$ is the logarithmic derivative of the conductivity with respect to the uniform generation rate,

$$
\gamma_{\sigma}=\partial\left[\ln \left(\sigma_{0}\right)\right] / \partial\left[\ln \left(G_{0}\right)\right] .
$$

$L_{n}, \quad L_{p}$ and $L_{a}$ are given by $L_{n}^{2}=k_{b} T \mu_{n} n_{0} /\left(q G_{0}\right), L_{p}^{2}=k_{b} T \mu_{p} p_{0} /\left(q G_{0}\right)$, and $L_{a}^{2}=$ $2 L_{n}^{2} L_{p}^{2} /\left(L_{n}^{2}+L_{p}^{2}\right)$. When the free-carrier concentrations under light are much higher than under dark, they are equal to the diffusion length of free-electrons, free-holes, and the ambipolar diffusion lengths of free-carriers, respectively [20]. $\tau_{d}$ is the dielectric relaxation time $\left(\tau_{d}=\varepsilon / \sigma_{0}\right), \tau_{p}$ and $\tau_{n}$ are given by $\tau_{p}=p_{0} / G_{0}$ and $\tau_{n}=n_{0} / G_{0}$, and they are respectively equal to the usual free-holes and free-electrons lifetimes when the free-carrier concentrations under light are much higher than under dark [20,21]. $L_{D}^{2}$ is only multiplied by $k^{2}$ in equation (12), thus is entirely related to the diffusion (D) of photocarriers. $L_{d}^{2}$ is multiplied by $\xi_{\text {ext }}^{2}$, therefore is related to the drift (d) of photocarriers. $L_{d D}^{2}$ multiplies $k^{2}$ in one term and $\xi_{\text {ext }}^{2}$ in another; thus, it may be linked to both processes (dD). From now on, we assume that the concentrations of photo-generated electrons and holes are much higher than their thermal equilibrium values. This assumption is fulfilled for the high generation rates of photocarriers used in this work.

A simple physical interpretation of why $\left|\Delta J / J_{0}\right|$ decreases with $k$ or $\xi_{\text {ext }}$ is given below [see equation (12)]. The interference pattern produces consecutive regions of high and low carrier concentrations in the material, i.e., the electron and hole carrier concentrations are given by $n=n_{0}+\Delta n_{0} \cos \left(k x+\varphi_{n}\right)$ and $p=p_{0}+\Delta p_{0} \cos \left(k x+\varphi_{p}\right)$, respectively. It can be proved that for a given temperature and light intensity,

$$
\left|\Delta J / J_{0}\right| \propto \Delta n_{0} \Delta p_{0} \frac{\sin \left(\varphi_{p}-\varphi_{n}\right)}{k \xi_{\text {ext }}}
$$


$\left|\Delta J / J_{0}\right|$ decreases when $\Delta n_{0}, \Delta p_{0}$ and $\frac{\sin \left(\varphi_{p}-\varphi_{n}\right)}{k \xi_{\text {ext }}}$ decreases, and each of these factors decreases with $k$ or $\xi_{\text {ext }}$. The third factor decreases with $k$ or $\xi_{\text {ext }}$ due to the presence of $k$ and $\xi_{\text {ext }}$ in the denominator. Although the main reduction of $\left|\Delta J / J_{0}\right|$ comes from $\Delta n_{0}$ and $\Delta p_{0}$. When $k$ increases, the period of the interference pattern $(\Lambda)$ decreases, and the regions of low and high carrier concentrations are now closer to each other. Consequently, some extra-carriers from the high concentration regions can diffuse toward the low concentration regions, decreasing the amplitudes of each photocarrier grating $\left(\Delta n_{0}\right.$ and $\left.\Delta p_{0}\right)$, and consequently $\left|\Delta J / J_{0}\right|$. When the applied electric field increases, the drift lengths of the photocarriers increase, and some extracarriers from the high-concentration regions can travel to the low-concentration regions, decreasing the amplitudes of each photocarrier grating $\left(\Delta n_{0}\right.$ and $\left.\Delta p_{0}\right)$ and, consequently decreasing $\left|\Delta J / J_{0}\right|$.

\subsection{Experimental configuration}

Figure 1 presents the basic experimental setup used in the SSPG technique [11]. The generation rate in the material [equation (1)] is produced by the interference of two monochromatic light beams of different intensities. When one of the beams arrives perpendicularly to the sample, as in figure 1 , the spatial period of the interference pattern $(\Lambda)$ is related to the angle between the incident beams $\delta$, by $\Lambda=\lambda / \sin (\delta)$, where $\lambda$ is the wavelength of the laser light in air. The generation rates induced by the strong $\left(G_{1}\right)$ or weak $\left(G_{2}\right)$ beams are related to $G_{0}$ and $\Delta G_{0}$ [see equation (1)] by the equations, $G_{0}=G_{1}+G_{2}$ and $\Delta G_{0}=2 \gamma_{0} \sqrt{G_{1} G_{2}}$. $\gamma_{0}$ is an interference quality factor: a positive number lower than 1 , taking into account the reduction of the light grating due to the partial coherence and polarization of the beams, light scattering, and mechanical vibrations [1].

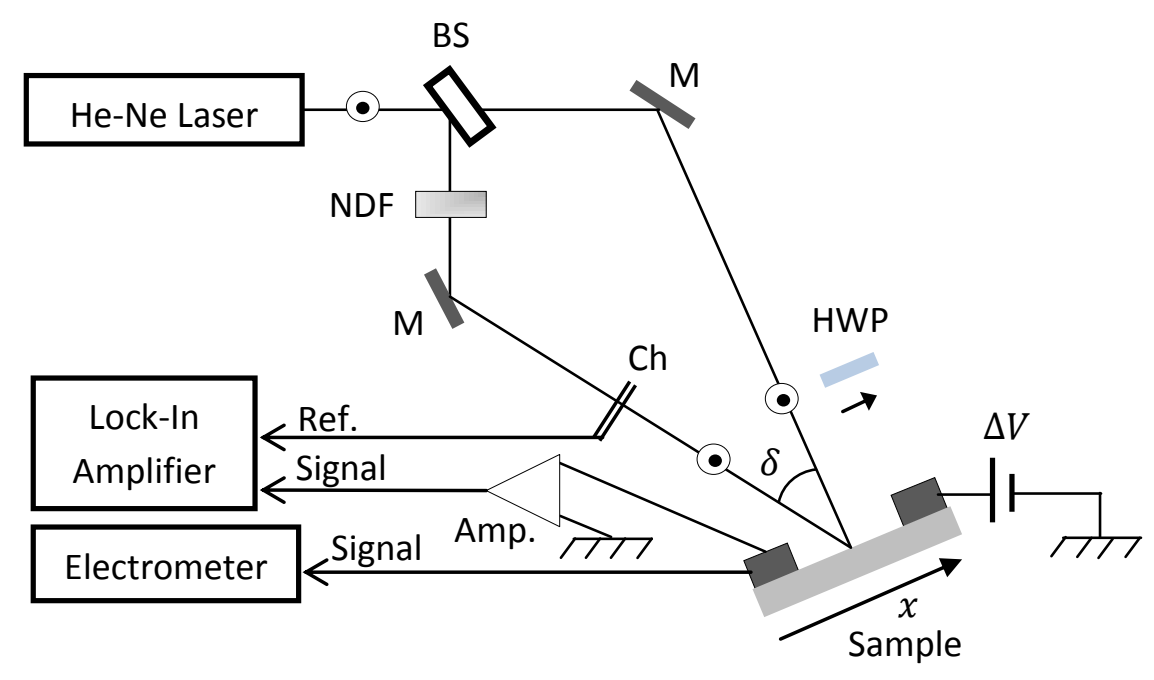

Figure 1. Experimental setup used in the SSPG technique. A linearly polarized laser beam is divided in two with a beam splitter (BS). Then one of the beams is attenuated with a neutral density filter (NDF) to obtain a large difference between their intensities. The beams are made to coincide on the sample region between contacts by using mirrors (M). The polarization of one of the beams can be rotated $90^{\circ}$ with a half-wave plate (HWP) to destroy the interference without changing the 
mean light intensity. A constant potential difference between the contacts is applied to induce a current, which can be measured with an electrometer. A lock-in amplifier is used to measure the alternating current induced in the material by chopping (Ch) the weak beam at a low frequency.

To obtain the decrease of the steady-state photocurrent by the light grating in relation to its initial value, $\Delta J / J_{0}$, we need to make two measurements. One is the photocurrent induced when the beams present the same polarization (parallel to the electrodes in the figure) and interfere with each other, $J_{0}+\Delta J$. The other is the photocurrent generated when the beams present perpendicular polarizations to each other and do not interfere, $J_{0}$. A half-wave plate (HWP) is generally used to rotate by $90^{\circ}$ the polarization of one of the beams. $\Delta J / J_{0}$ can be obtained from,

$$
\frac{\Delta J}{J_{0}}=\frac{\left(J_{0}+\Delta J\right)}{J_{0}}-1
$$

It is possible to increase the precision of the acquisition by chopping the weak beam at a low frequency and measuring, with a lock-in amplifier, the alternating current induced when the beams interfere or not. The quotient between these two quantities, $\beta$, is related to $\Delta J$ by [1],

$$
\beta=\frac{J_{0}+\Delta J-J_{1}}{J_{0}-J_{1}}=1+\frac{\Delta J}{\left(\sigma_{0}-\sigma_{1}\right) \xi_{\text {ext }}}
$$

where $J_{1}=\sigma_{1} \xi_{\text {ext }}$ is the direct current induced when only the more intense beam illuminates the sample. Replacing equation (12) in (21) we obtain:

$$
\beta=1-\frac{\varphi_{0}^{2}\left[1+L_{d D}^{4} k^{2} / L_{0}^{2}\right]}{\left\{1+\left[L_{D}^{2}+\frac{L_{d D}^{4}}{2}\left\{k^{2}+\left(\frac{q \xi_{\text {Ext }}}{k_{b} T}\right)^{2}\right\}\right] k^{2}\right\}^{2}+\left(\frac{q \xi_{\text {Ext }}}{k_{b} T}\right)^{2} L_{d}^{4} k^{2}},
$$

where $\varphi_{0}^{2}$ is given by,

$$
\varphi_{0}^{2}=\frac{\gamma_{\sigma}^{2} \Delta G_{0}^{2} \sigma_{0}}{2 G_{0}^{2}\left(\sigma_{0}-\sigma_{1}\right)} \simeq \frac{2 \gamma_{0}^{2} \gamma_{\sigma}}{1+G_{2} / G_{1}} .
$$

Note that obtaining $\beta$ is equivalent to finding $\Delta J / J_{0} . \beta$ (or $\Delta J / J_{0}$ ) is a function of only three unknowns: $L_{D}^{2}, L_{d}^{2}$ and $L_{d D}^{2}$; because $L_{0}^{2}$ and $\varphi_{0}^{2}\left(\theta_{0}^{2}\right)$ can be estimated from the SSPC. Expression (22) is more compact than the one presented by Abel et al. [5], which is a function of 5 unknowns. We have reduced the number of unknowns by combining the general expression with the differential neutrality and continuity equations.

\subsection{RZW equation}

The terms $L_{d D}^{4} k^{2} / L_{0}^{2}$ in the numerator of equation (22) and the term $L_{d D}^{4} k^{2} / 2$ in its denominator can be neglected for low enough $k^{2}$ values. Making $\xi_{\text {ext }} \rightarrow 0$, the equation reduces to the well-known RZW formula [1],

$$
\beta \simeq 1-\frac{\varphi_{0}^{2}}{\left(1+L_{D}^{2} k^{2}\right)^{2}}
$$


which presents just two fitting parameters, $\varphi_{0}^{2}$ and $L_{D}^{2}$. The first term of $L_{D}^{2}$ [see equation (15)] is the square of the ambipolar diffusion length, $L_{a m b}^{2}=\left(\gamma_{n}+\gamma_{p}\right) L_{a}^{2} / 2$ [22]. As was pointed out before [6], the RZW formula could only be used for the estimation of $L_{a m b}$ when the two last terms of equation (15) are negligible (i.e., in the "lifetime regime"). Similar results were obtained using the phenomenological model that does not distinguish between trapped and free charges [2-4]. The first term of expression (15) coincides with the results obtained with the phenomenological model, whereas the other two present differences. The reason comes from the fact that one of the assumptions of the phenomenological model is not sound within the MT model. It assumes that the electron (hole) drift mobility is only a function of the total electron (hole) concentration. However, from its definition, it can be shown that, within the MT model, it is also a function of the total hole (electron) concentration. The analytical proof can be found in Appendix B.

According to this new result, we should consider the phenomenological model as an oversimplification of the MT model, even under steady-state conditions. It can be observed with numerical simulations that the previous methods for correcting the overestimation of $L_{a m b}$ in the relaxation regime [2-4] do not work properly.

\subsection{Extrinsic semiconductors}

When the mobility-concentration product of one type of carrier is much larger than that of the other, as it usually happens, additional simplifications can be made. From now on, we assume that the material is n-type $\left(\mu_{n} n_{0} \gg \mu_{p} p_{0}\right)$, but the equations for a p-type material can be obtained, replacing $n$ with $p$ and vice versa in the final formulas.

For an n-type material, $\sigma_{0} \simeq q \mu_{n} n_{0}, \gamma_{n} \simeq \gamma_{\sigma}, L_{a}^{2} \simeq 2 L_{p}^{2}, L_{0}^{2} \simeq \gamma_{n} L_{n}^{2}$ and generally $\mu_{n} \tau_{n}^{r} \gg \mu_{p} \tau_{p}^{r}$. The last inequality allows us to discard the third term in front of the second term in equations (15) and (17), which become respectively,

$$
\begin{aligned}
& L_{D}^{2} \simeq\left(\gamma_{n}+\gamma_{p}\right) L_{p}^{2}+\frac{\tau_{d} \gamma_{n} L_{n}^{2}}{\left(1+Q^{+}\right) \tau_{p}^{r}} \\
& L_{d}^{2} \simeq\left(\gamma_{n}-\gamma_{p}\right) L_{p}^{2}+\frac{\tau_{d} \gamma_{n} L_{n}^{2}}{\left(1+Q^{+}\right) \tau_{p}^{r}}
\end{aligned}
$$

The condition $\mu_{n} n_{0} \gg \mu_{p} p_{0}$ and $\mu_{n} \tau_{n}^{r} \gg \mu_{p} \tau_{p}^{r}$ are clearly satisfied in an unintentionally-doped aSi:H-like material. Therefore, the previous equations are excellent approximations in this case. It was observed in the numerical simulations that for this kind of material, $L_{d}$ is real because the absolute value of the first term of expression (26) is always lower than the second one. The bipolarity of the transport is evident in equations (25) and (26), where the first terms are proportional to the minority carrier diffusion length, whereas the second ones are proportional to the majority carrier diffusion length.

\subsection{Equations for $\gamma_{p}$ and $L_{p}^{2}$}


$\gamma_{p}$ can be estimated from the hole diffusion lengths measured at two nearby generation rate values, according to the following formula obtained from the definitions of $L_{p}^{2}$ and $\gamma_{p}$,

$$
\gamma_{p}=1+\frac{\partial\left[\ln \left(L_{p}^{2}\right)\right]}{\partial\left[\ln \left(G_{0}\right)\right]}
$$

We can get rid of the second term of equation (25) by subtracting equation (26), to obtain an equation for $L_{p}^{2}$,

$$
L_{p}^{2} \simeq \frac{L_{D}^{2}-L_{d}^{2}}{2 \gamma_{p}}
$$

As we will see in the next section, it is not easy to estimate precisely $L_{d}^{2}$ from the experimental data. It is necessary to find another equation for $L_{p}^{2}$ that is a function of parameters that can be measured more precisely.

The second terms of the right-hand side of equations (25) and (26) can be written as a function of $L_{p}^{2}$ using equation (16),

$$
\frac{\tau_{d} \gamma_{n} L_{n}^{2}}{\left(1+Q^{+}\right) \tau_{p}^{r}}=\frac{\tau_{p} L_{d D}^{4}}{2 \tau_{p}^{r} L_{p}^{2}}
$$

Replacing equation (29) in equation (25) or (26), and using equation (28) we obtain the following quadratic equation for $L_{p}^{2}$,

$$
\left(\gamma_{n}+\gamma_{p}-2 \gamma_{p}^{2} \frac{\tau_{p}}{\tau_{p}^{r}}\right) L_{p}^{4}-\left(1-2 \gamma_{p} \frac{\tau_{p}}{\tau_{p}^{r}}\right) L_{D}^{2} L_{p}^{2}+\frac{\tau_{p}}{2 \tau_{p}^{r}}\left(L_{\xi}^{4}-L_{D}^{4}\right) \simeq 0,
$$

where $L_{\xi}^{4}$ is defined as,

$$
L_{\xi}^{4}=L_{d D}^{4}+L_{d}^{4}
$$

The solution of equation (30) is given by,

$$
L_{p}^{2} \simeq \frac{\left(1-2 \gamma_{p} \frac{\tau_{p}}{\tau_{p}^{p}}\right) L_{D}^{2}+\sqrt{\left[1+2\left(\gamma_{n}-\gamma_{p}\right) \frac{\tau_{p}}{\tau_{p}^{r}}\right] L_{D}^{4}-2 \frac{\tau_{p}}{\tau_{p}^{p}}\left(\gamma_{n}+\gamma_{p}-2 \gamma_{p}^{2} \frac{\tau_{p}}{\tau_{p}^{p}}\right) L_{\xi}^{4}}}{2\left[\gamma_{n}+\gamma_{p}-2 \gamma_{p}^{2} \frac{\tau_{p}}{\tau_{p}^{p}}\right]} .
$$

Further simplifications can be made assuming the high doping condition, $c_{n}(E) n_{0} \gg c_{p}(E) p_{0}$, where $c_{n}(E)$ and $c_{p}(E)$ are the electron and hole capture coefficients of the localized states with energy $E$. In Appendix $C$ we show that, in this case, $\tau_{p}^{r} \simeq \tau_{p}$ and $\gamma_{p}<1$. The first relation allows us to replace $\tau_{p} / \tau_{p}^{r}$ by one in equation (32), which becomes in this way in a recursive formula for the estimation of $L_{p}^{2}$ from parameters that can be directly measured,

$$
L_{p}^{2} \simeq \frac{\left(1-2 \gamma_{p}\right) L_{D}^{2}+\sqrt{\left[1+2\left(\gamma_{n}-\gamma_{p}\right)\right] L_{D}^{4}-2\left(\gamma_{n}+\gamma_{p}-2 \gamma_{p}^{2}\right) L_{\xi}^{4}}}{2\left(\gamma_{n}+\gamma_{p}-2 \gamma_{p}^{2}\right)} .
$$

According to numerical calculations, $\gamma_{n}$ and $\gamma_{p}$ present a much lower variation with the generation rate than $L_{D}^{2}$ and $L_{\xi}^{4}$. Thus, we can assume that they are constant for two nearby 
generation rate values. $\gamma_{n}$ can be obtained from SSPC using equation (18). Although, another equation for estimating $\gamma_{n}$ from $\varphi_{0}$ can be obtained from equation (23) $[6,23,24]$,

$$
\gamma_{n} \simeq \gamma_{\sigma}=\frac{\left(1+G_{2} / G_{1}\right)}{2 \gamma_{0}^{2}} \varphi_{0}^{2}
$$

Before using equation (34), it is necessary to estimate the constant $\left(1+G_{2} / G_{1}\right) /\left(2 \gamma_{0}^{2}\right)$, which neither depends on $T$ nor $G_{0}$. It can be done by estimating $\gamma_{n}$ from SSPC for at least one temperature and generation rate, at which we have also measured $\varphi_{0}^{2}$.

In order to use equation (33), we first need to measure $L_{D}^{2}, L_{\xi}^{4}$ and $\sigma_{0}$ or $\varphi_{0}$, for two nearby generation rate values. $\gamma_{n}$ is then estimated from $\sigma_{0}$ or from $\varphi_{0}$. Assigning to $\gamma_{p}$ the same arbitrary value lower than 1 for both generation rates, we obtain the first approximation of $L_{p}^{2}$ for each generation rate value with formula (33). The initial estimate of $\gamma_{p}$ is then corrected by replacing the $L_{p}^{2}$ and $G_{0}$ values in equation (27). Replacing the new estimate of $\gamma_{p}$ in equation (33), we correct the initial calculations of $L_{p}^{2}$, and we continue with this procedure until convergence. The following section presents the best ways of obtaining $\varphi_{0}, L_{D}^{2}$, and $L_{\xi}^{4}$ experimentally.

The high doping condition, $c_{n}(E) n_{0} \gg c_{p}(E) p_{0}$, is not strictly verified for all the temperatures in the unintentionally-doped a-Si:H-like material simulated in section 3 . As the temperature increases, the ratio $n_{0} / p_{0}$ decreases due to a greater influence of the dangling bonds states [25], producing slight underestimations in the $L_{p}^{2}$ values extracted from equation (33) (see subsection 3.3).

\subsection{Extraction of $\varphi_{0}, L_{D}^{2}$, and $L_{\xi}^{4}$ from the experimental data}

A reasonable approximation of $\beta$ for small $k$ values is obtained by discarding the second term inside the numerator square bracket in equation (22). Expressing $\beta$ explicitly as function of $\left[q \xi_{\text {ext }} /\left(k_{b} T\right)\right]^{2}$, it is obtained,

$$
\beta \simeq 1-\frac{1}{\varphi_{k}^{-2}+L_{k}^{2}\left(\frac{q \xi_{e x t}}{k_{b} T}\right)^{2}+\frac{L_{d D}^{8} k^{4}}{4 \varphi_{0}^{2}}\left(\frac{q \xi_{\text {ext }}}{k_{b} T}\right)^{4}}
$$

where $\varphi_{k}^{-2}$ and $L_{k}^{2}$ are given by,

$$
\begin{aligned}
\varphi_{k}^{-2} & =\frac{1+2 L_{D}^{2} k^{2}+\left(L_{D}^{4}+L_{d D}^{4}\right) k^{4}}{\varphi_{0}^{2}} \\
L_{k}^{2} & =\left(L_{\xi}^{4}+L_{D}^{2} L_{d D}^{4} k^{2}\right) \frac{k^{2}}{\varphi_{0}^{2}}
\end{aligned}
$$

Using a numerical simulation with a-Si:H parameters, we observed that the last term inside the denominator of equation (35) is negligible for the electric field values that we can achieve experimentally $\left(\xi_{\text {ext }} \leq 5000 \mathrm{~V} \mathrm{~cm}^{-1}\right.$ ), especially for $k$ values that are not extremely high. In this case, equation (35) becomes, 


$$
(1-\beta)^{-1} \simeq \varphi_{k}^{-2}+L_{k}^{2}\left(\frac{q \xi_{\text {ext }}}{k_{b} T}\right)^{2}
$$

The plot of the experimental data, $(1-\beta)^{-1}$ vs $\left[q \xi_{\text {ext }} /\left(k_{b} T\right)\right]^{2}$ for each $k$ value, can be fitted with the previous linear equation to obtain $\varphi_{k}^{-2}$ and $L_{k}^{2}$. When we have plotted the experimental values of $\varphi_{k}^{-2}$ vs $k^{2}$ and $L_{k}^{2}$ vs $k^{2}$ we observed a parabolic behavior, as predicted by equations (36) and (37), respectively. Nevertheless, a strange behavior was observed for $L_{k}^{2}$ vs $k^{2}$ in certain samples: the intercept with the ordinate axis was clearly different from zero. A better fit of the experimental data was obtained by adding an arbitrary constant $\pm L_{S}^{2}$ to equation (37),

$$
L_{k}^{2} \simeq \frac{ \pm L_{S}^{2}+L_{\xi}^{4} k^{2}+\left[L_{D}^{2}\left(L_{\xi}^{4}-L_{d}^{4}\right)\right] k^{4}}{\varphi_{0}^{2}}
$$

We think that $L_{S}^{2}$ may be related to slight non-Ohmicities in the electrical behavior of the contacts, which have not been considered in our model (see subsection 4.1).

Using a numerical simulation, we can test the precision of equations (36) and (39) for fitting the values of $\varphi_{k}^{-2}$ and $L_{k}^{2}$ obtained from the previous fit of the data with equation (38). The $k^{4}$ terms in equations (36) and (39) always present larger errors than the other lower-order terms because equation (38) becomes more exact as $k$ decreases. Another problem of equations (36) and (39) is that they present many independent fitting parameters, which produces inconsistent fittings of the experimental data due to the experimental errors. In order to make more confident fits, we can reduce the number of fitting parameters by approximating the $k^{4}$ terms.

Discarding $L_{d D}^{4}$ in front of $L_{D}^{4}$ in equation (36), we obtain a formula equivalent to the RZW equation,

$$
\varphi_{k}^{-1} \simeq \frac{\left(1+L_{D}^{2} k^{2}\right)}{\varphi_{0}}
$$

from which we can obtain good estimates of $L_{D}^{2}$ and $\varphi_{0}$. In order to approximate $L_{d}^{4}$ in equation (39) we combine equation (28) with equation (33) to obtain,

$$
L_{d}^{2} \simeq \frac{\gamma_{n} L_{D}^{2}-\gamma_{p} \sqrt{\left[1+2\left(\gamma_{n}-\gamma_{p}\right)\right] L_{D}^{4}-2\left(\gamma_{n}+\gamma_{p}-2 \gamma_{p}^{2}\right) L_{\xi}^{4}}}{\left(\gamma_{n}+\gamma_{p}-2 \gamma_{p}^{2}\right)} .
$$

Another much simpler equation for $L_{d}^{2}$ is obtained by combining equations (26), (28) and (29), and making the approximations $\tau_{p} / \tau_{p}^{r} \sim 1$ and $\gamma_{n} \simeq \gamma_{p} \sim 1$,

$$
L_{d}^{2} \simeq \frac{L_{\xi}^{4}}{L_{D}^{2}}
$$

Replacing expressions (41) or (42) into equation (39), we respectively obtain,

$$
L_{k}^{2} \simeq \frac{ \pm L_{S}^{2}+L_{\xi}^{4} k^{2}+\left\{L_{D}^{2}\left[L_{\xi}^{4}-\left(\frac{\gamma_{n} L_{D}^{2}-\gamma_{p} \sqrt{\left[1+2\left(\gamma_{n}-\gamma_{p}\right)\right] L_{D}^{4}-2\left(\gamma_{n}+\gamma_{p}-2 \gamma_{p}^{2}\right) L_{\xi}^{4}}}{\left(\gamma_{n}+\gamma_{p}-2 \gamma_{p}^{2}\right)}\right)^{2}\right]\right\} k^{4}}{\varphi_{0}^{2}},
$$




$$
L_{k}^{2} \simeq \frac{ \pm L_{S}^{2}+L_{\xi}^{4} k^{2}+L_{D}^{2} L_{\xi}^{4}\left(1-\frac{L_{\xi}^{4}}{L_{D}^{4}}\right) k^{4}}{\varphi_{0}^{2}} .
$$

$\gamma_{p}$ in equation (43) can be estimated from,

$$
\gamma_{p} \simeq 1+\frac{\partial\left[\ln \left(L_{D}^{2}\right)\right]}{\partial\left[\ln \left(G_{0}\right)\right]}
$$

which was obtained by replacing equation (28) in equation (27) and discarding $L_{d}^{2}$. Fitting the experimental values of $L_{k}^{2}$ vs $k^{2}$ with equation (43) or (44) we can obtain $L_{\xi}^{4}$ and $\pm L_{S}^{2}$. Using a numerical simulation of an unintentionally-doped a-Si:H-like material, we present an evaluation of the results obtained with each of the equations in subsection 3.3; equation (43) is just slightly more precise than equation (44) at very high temperatures. Experimentally, we were not able to observe any difference between the results obtained with each of the equations.

\subsection{Equation for $\varepsilon c_{n}^{r} /\left(q \mu_{n}\right)$}

We define a new recombination lifetime related to the totality of holes (free plus trapped),

$$
\tau_{p}^{t}=\left(1+Q^{+}\right) \tau_{p}^{r} \simeq Q^{+} \tau_{p}^{r}
$$

The approximate equality is valid for materials with a large density of localized states such as amorphous materials, where $Q^{+} \gg 1$. Replacing the expressions for $Q^{+}$[equation (A12)] and $\tau_{p}^{r}$ [equation (A16)] in the previous equality we obtain,

$$
\tau_{p}^{t} \simeq \frac{\int_{E_{v}}^{E_{c}} c_{p}(E)\left[c_{n}(E) n_{0}+e_{p}(E)\right] \tau^{2}(E) N(E) \mathrm{d} E}{\int_{E_{v}}^{E_{c}}\left[c_{n}(E) n_{0}+e_{n}(E)\right] c_{p}(E)\left[c_{n}(E) n_{0}+e_{p}(E)\right] \tau^{2}(E) N(E) \mathrm{d} E} .
$$

In a a-Si:H-like material, the second term inside the denominator's first parenthesis $\left[e_{n}(E)\right]$ can be neglected in front of the first one. Assuming also that the electron capture coefficient is independent of the energy, $c_{n}(E)=c_{n}^{r}$, it is obtained,

$$
\tau_{p}^{t} \simeq\left(c_{n}^{r} n_{0}\right)^{-1}
$$

In this case, $\tau_{d} / \tau_{p}^{t}$ becomes a constant given by [6],

$$
\frac{\tau_{d}}{\tau_{p}^{t}}=\frac{\tau_{d}}{\left(1+Q^{+}\right) \tau_{p}^{r}} \simeq \frac{\varepsilon c_{n}^{r}}{q \mu_{n}}
$$

In subsection 3.2, we show that when the electron capture coefficient depends on the state's energy, $\tau_{d} / \tau_{p}^{t}$ is not constant and depends on the temperature and generation rate. In this case, $c_{n}^{r}$ corresponds to an average of the recombination states' electron capture coefficient [26].

Replacing equation (49) into equation (25), we obtain the following formula for the estimation of $\varepsilon c_{n}^{r} /\left(q \mu_{n}\right)$,

$$
\frac{\varepsilon c_{n}^{r}}{q \mu_{n}} \simeq \frac{L_{D}^{2}-\left(\gamma_{n}+\gamma_{p}\right) L_{p}^{2}}{\gamma_{n} L_{n}^{2}}
$$

where $L_{n}^{2}$ can be estimated from the following formula, 


$$
L_{n}^{2} \simeq\left(\frac{k_{b} T}{q}\right) \frac{\sigma_{0}}{q G_{0}} .
$$

Due to the subtraction in equation (50), the relative errors expected for $\varepsilon c_{n}^{r} /\left(q \mu_{n}\right)$ are higher than those obtained for $L_{D}^{2}$ or $\left(\gamma_{n}+\gamma_{p}\right) L_{p}^{2}$, especially when approaching the lifetime regime where $L_{D}^{2} \simeq\left(\gamma_{n}+\gamma_{p}\right) L_{p}^{2}$.

\subsection{Validity of the low-voltage approach}

The low-voltage SSPG estimates the ambipolar diffusion length, $L_{a m b}^{2}=\left(\gamma_{n}+\gamma_{p}\right) L_{p}^{2}$, by fitting the experimental values of $\beta$ vs $k^{2}$ for a low applied electric field with the RZW formula [equation (24)] [1,11]. The $L_{a m b}^{2}$ relative errors obtained from this approach in an n-type semiconductor can be estimated from equation (25),

$$
E_{R}\left[L_{a m b}^{2}\right]=\frac{L_{D}^{2}-L_{a m b}^{2}}{L_{a m b}^{2}} \simeq \frac{\gamma_{n}}{\left(\gamma_{n}+\gamma_{p}\right)} \frac{\varepsilon c_{n}^{r}}{q \mu_{n}} \frac{L_{n}^{2}}{L_{p}^{2}} .
$$

The right-hand side of the equation can also be written as,

$$
E_{R}\left[L_{a m b}^{2}\right] \simeq \frac{\gamma_{n}}{\left(\gamma_{n}+\gamma_{p}\right)} \frac{\tau_{p}^{\sigma}}{\tau_{p}^{t}}
$$

where $\tau_{p}^{\sigma}$ is defined similarly to the dielectric relaxation time, but instead of being inversely proportional to the conductivity, it is inversely proportional to the minority carrier contribution to the conductivity,

$$
\tau_{p}^{\sigma}=\frac{\varepsilon}{\sigma_{p}}=\frac{\varepsilon}{q \mu_{p} p_{0}}
$$

According to equation (53), the low-voltage SSPG is only valid when the experimental conditions guarantee that $\tau_{p}^{t} \gg \tau_{p}^{\sigma}$. This condition is stricter and more precise than $\tau_{p}^{t} \gg \tau_{d}$, previously suggested as a candidate for defining the range of validity of the RZW formula (i.e., the lifetime regime) [6]. After making the approximation $\gamma_{n} /\left(\gamma_{n}+\gamma_{p}\right) \sim 1 / 2$, we can directly estimate $E_{R}\left[L_{a m b}^{2}\right]$ from the ratio $\tau_{p}^{\sigma} / \tau_{p}^{t}$. For example, $\tau_{p}^{\sigma} / \tau_{p}^{t}=1$ implies a $\sim 50 \%$ error in the estimation of $L_{a m b}^{2}$ from the low-voltage SSPG technique.

\section{Numerical simulation}

\subsection{Procedure}

This section tests the precision of the previously presented formulas and procedures using a numerical simulation of an unintentionally-doped a-Si:H-like material. We also detail the sources of errors and clarify some of the concepts and procedures introduced previously. To simulate the photoconductive behavior of the material, we use the following donor-like and acceptor-like densities of localized states per unit energy:

$$
N^{D O N}(E)=N\left(E_{V}\right) \exp \left(\frac{E_{V}-E}{k_{b} T_{V}}\right)+\frac{N_{\delta}}{\sqrt{2 \pi} W_{\delta}} \exp \left(\frac{-\left(E-E_{\delta}\right)^{2}}{2 W_{\delta}^{2}}\right),
$$




$$
N^{A C C}(E)=N\left(E_{C}\right) \exp \left(\frac{E-E_{C}}{k_{b} T_{C}}\right)+\frac{N_{\alpha}}{\sqrt{2 \pi} W_{\alpha}} \exp \left(\frac{-\left(E-E_{\alpha}\right)^{2}}{2 W_{\alpha}^{2}}\right)
$$

The first terms correspond to exponential band tails originating from the dispersion of bond angles and bond lengths due to the long-range disorder in the atomic lattice. The second terms correspond to deep Gaussian states coming from coordination defects (mostly dangling bonds) $[18,19,25]$. Table 1 presents the values of the material parameters used in this simulation, which correspond to a device-quality material $[7,19,27,28]$.

Table 1. Material parameters used in the numerical simulation, corresponding to an unintentionally-doped a-Si:H-like material.

\begin{tabular}{ll}
\hline \hline Parameter & Value \\
\hline$c_{n}, c_{p}$ (band tails) $\left[\mathrm{cm}^{3} \mathrm{~s}^{-1}\right]$ & $3 \times 10^{-9}$ \\
$c_{n}, c_{p}$ (deep states) $\left[\mathrm{cm}^{3} \mathrm{~s}^{-1}\right]$ & $3 \times 10^{-8}$ \\
$\mu_{n}\left[\mathrm{~cm}^{2} \mathrm{~V}^{-1} \mathrm{~s}^{-1}\right]$ & 10 \\
$\mu_{p}\left[\mathrm{~cm}^{2} \mathrm{~V}^{-1} \mathrm{~s}^{-1}\right]$ & 1 \\
$\varepsilon\left[\mathrm{F} \mathrm{cm}^{-1}\right]$ & $1.06 \times 10^{-12}$ \\
$N\left(E_{V}\right), N\left(E_{C}\right)\left[\mathrm{cm}^{-3} \mathrm{eV}^{-1}\right]$ & $2 \times 10^{21}$ \\
$N_{\alpha}, N_{\delta}\left[\mathrm{cm}^{-3} \mathrm{eV}^{-1}\right]$ & $1 \times 10^{16}$ \\
$W_{\alpha}, W_{\delta}[\mathrm{eV}]$ & 0.3 \\
$E_{\delta}[\mathrm{eV}]$ & 0.9 \\
$E_{\alpha}[\mathrm{eV}]$ & 1.15 \\
$E_{C}-E_{V}[\mathrm{eV}]$ & 1.8 \\
$T_{C}[\mathrm{~K}]$ & 240 \\
$T_{V}[\mathrm{~K}]$ & 600 \\
\hline \hline
\end{tabular}

The results presented in this section were obtained using the following procedure. For given $T$ and $G_{0}$ values, we calculate the uniform concentrations of free carriers, $n_{0}$ and $p_{0}$, by simultaneously solving the continuity and the charge neutrality equations [(A1) and (A2), respectively]. The same is done for a slightly lower value of the generation rate, $G_{1}$. For given $k$ and $\xi_{\text {ext }}$ values, we use the previously calculated values to compute $\beta$ from equation (22).

\subsection{Evaluation of the low-voltage approach}

Figure 2 presents the diffusion length squared obtained from the RZW equation $\left(L_{R Z W}^{2}\right)$ for different temperatures under a low applied voltage. $L_{R Z W}^{2}$ is then the value of $L_{D}^{2}$ obtained from the fitting of $\beta$ vs $k^{2}$ with equation (24). We have calculated $\beta$ for seven $\Lambda$ values similar to those obtained experimentally $(1.1 \mu \mathrm{m} \leq \Lambda \leq 9.0 \mu \mathrm{m})$, using a low external electric field of $500 \mathrm{~V} \mathrm{~cm}^{-1}$ and a typical uniform generation rate of $G_{0}=10^{20} \mathrm{~cm}^{-3} \mathrm{~s}^{-1}$. In order to make a comparison, we also have plotted the exact values of $L_{D}^{2}$ and $L_{a m b}^{2}$ for the same conditions. 


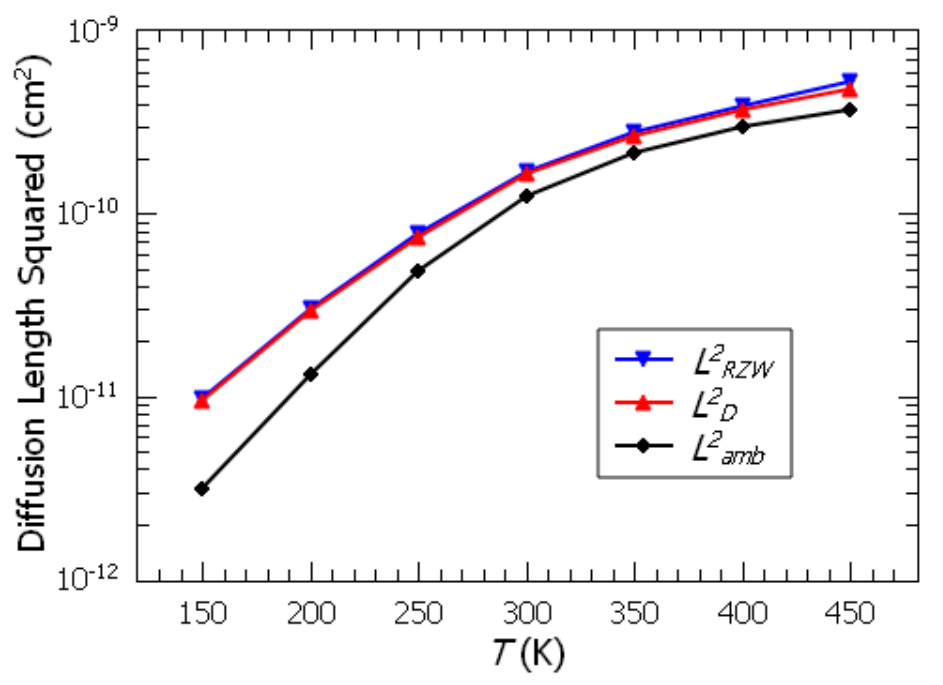

Figure 2. Diffusion length squared vs temperature obtained for $G_{0}=10^{20} \mathrm{~cm}^{-3} \mathrm{~s}^{-1}$. The down triangles were obtained by fitting the data with the RZW formula. $L_{D}^{2}$ corresponds to the general expression of the diffusion length given by equation (15), while $L_{a m b}^{2}$ is the ambipolar diffusion length [i.e., just the first term of equation (15)].

As expected, $L_{R Z W}^{2}$ overestimates $L_{a m b}^{2}$, and the overestimation tends to increase as the temperature decreases. Above room temperature, the overestimation does not reach $41 \%$, but at the lowest temperature, it exceeds $200 \%$. Some papers $[6,16,29,30]$ make the approximation $\gamma_{n}+\gamma_{p} \sim 2$ in order to determine the minority carrier diffusion length from the ambipolar diffusion length, $L_{p}^{2}=L_{a m b}^{2} /\left(\gamma_{n}+\gamma_{p}\right) \simeq L_{a m b}^{2} / 2$, which is an extra but generally a lower source of errors [11].

Figure 3 presents the three dimensionless factors of equation (52) for the same conditions, whose product gives the relative errors for $L_{a m b}^{2}$ obtained from the low-voltage SSPG, $E_{R}\left[L_{a m b}^{2}\right]$. It can be observed that the factor $L_{n}^{2} / L_{p}^{2}$ is the only one responsible for the increase of $E_{R}\left[L_{a m b}^{2}\right]$ with the decrease of temperature. The factor $\varepsilon c_{n}^{r} /\left(q \mu_{n}\right)$ presents a lower variation with temperature and in the opposite direction, and $\gamma_{n} /\left(\gamma_{n}+\gamma_{p}\right)$ is practically temperatureindependent compared to the other two factors. 


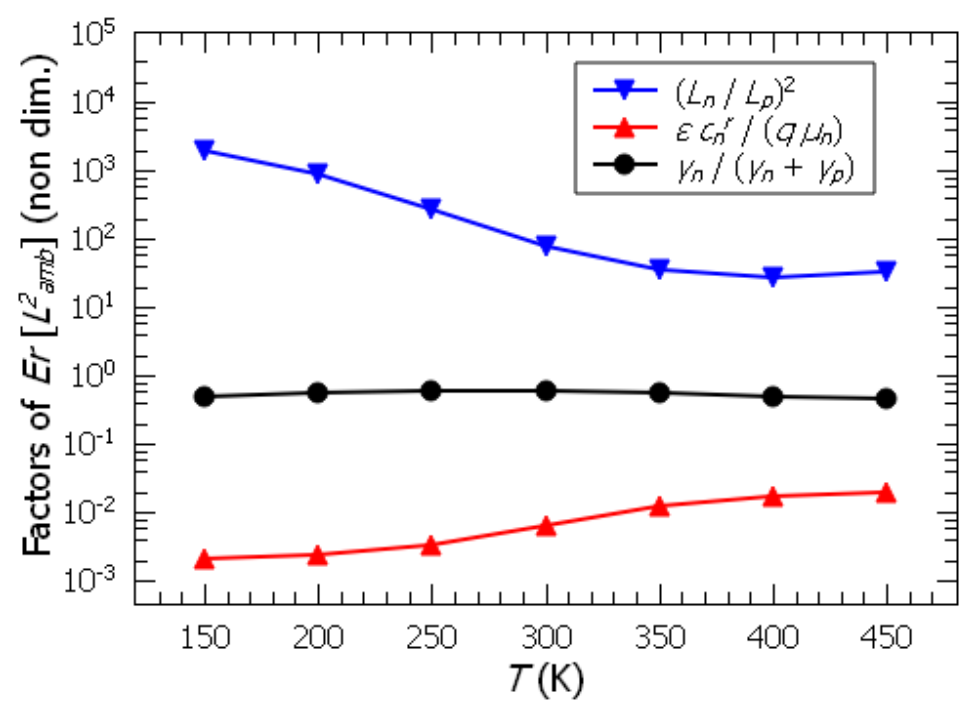

Figure 3. Dimensionless factors of equation (52), the product of which is equal to the relative error obtained for $L_{a m b}^{2}$ when using the low-voltage SSPG for its estimation.

To deduce equation (52), we used equation (49), which was obtained assuming that the electron capture coefficient is independent of the localized state's energy. This condition is not verified in some a-Si:H-like materials $[7,27,29]$, such as in the one we are simulating, where the electron capture coefficients of the deep states are higher than those from the band tail states (see table 1). Consequently, we observe an increase of $\varepsilon c_{n}^{r} /\left(q \mu_{n}\right)$ with temperature. Using the electron capture coefficient of the band tails states, we obtain $\varepsilon c_{n} /\left(q \mu_{n}\right)=1.99 \times 10^{-3}$; using the electron capture coefficient of the deep states, we find $\varepsilon c_{n} /\left(q \mu_{n}\right)=1.99 \times 10^{-2}$. In figure 3 we see that at low temperature, $\varepsilon c_{n}^{r} /\left(q \mu_{n}\right)$ approaches the first value and, at high temperature, approaches the second value. Decreasing the temperature produces a displacement of the trapped-carriers quasi-Fermi levels towards the mobility edges and, consequently, increases the recombination states density. As was pointed out by Tran [27], in a a-Si:H-like material, the valence band tail states are the principal responsible for the recombination at low temperatures. In contrast, the deep states are responsible for the recombination at high temperatures. Due to their low concentration, the influence of the conduction band tail states is neglected by this author. In other words, the lower the temperature, the higher the recombination states from the valence band tail compared to the deep states, and vice versa. Thus, the electron capture coefficient $c_{n}^{r}$, inside the previous formulas, corresponds to the average electron capture coefficient of the recombination states, i.e., those states located between the quasi-Fermi levels of trapped carriers [26]. A further discussion on the energy dependence of the capture coefficients will be presented elsewhere.

On the other hand, and in accordance with equation (24) and subsection 2.3, we observe in figure 2 that $L_{R Z W}^{2}$ provides a very good estimate of $L_{D}^{2}$. It becomes just slightly worst with temperature because the terms that were discarded in the deduction of the RZW equation increase more with temperature than the others. The other parameter that we can extract from the low-voltage SSPG is $\varphi_{0}^{2}$, which can be used for the estimation of $\gamma_{\sigma}$ according to equation 
(34). Figure 4 presents the values of $\gamma_{\sigma}$ obtained from $\varphi_{0}^{2}$ (SSPG), and the values obtained from equation (18) by calculating $\sigma_{0}$ for a $33 \%$ higher and $33 \%$ lower $G_{0}$ value. It is observed an excellent agreement between the two different ways of estimating $\gamma_{\sigma}$. In both cases, the relative errors of $\gamma_{\sigma}$ are not higher than $1 \%$.

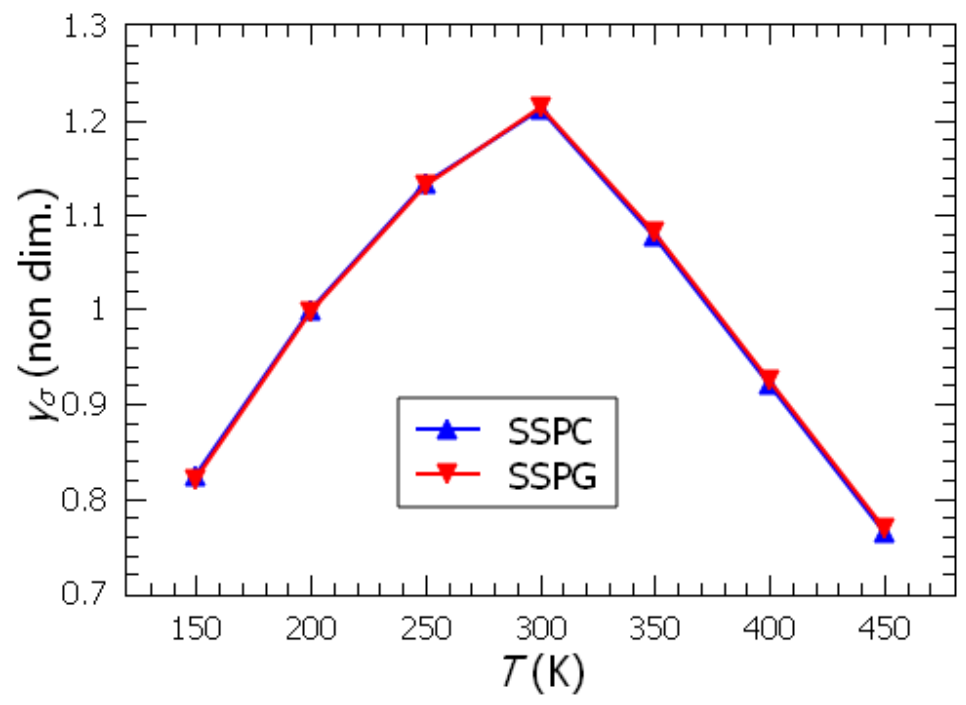

Figure 4. $\gamma_{\sigma}$ calculated at different temperatures. The up triangles were obtained by calculating $\sigma_{0}$ for two values of $G_{0}$ (SSPC) and using equation (18). The down triangles were obtained from the value of $\varphi_{0}^{2}$, previously found from the RZW equation, and using equation (34).

\subsection{Evaluation of the multi-voltage approach}

In figure 5, we plot the values of $L_{p}^{2}$ obtained from equation (33) and the exact ones for different temperatures and a typical generation rate of $G_{0}=10^{20} \mathrm{~cm}^{-3} \mathrm{~s}^{-1}$. It can be observed that with equation (33) we obtain an excellent estimation of $L_{p}^{2}$. The slight underestimation observed at the highest temperatures is due to the decrease of the ratio $L_{n}^{2} / L_{p}^{2}$ with temperature (see figure 3). The approximate equations (25), (26), and especially $\tau_{p}^{r} \simeq \tau_{p}$, from which equation (33) was deduced, become less precise as the ratio $n_{0} / p_{0}$ decreases. 


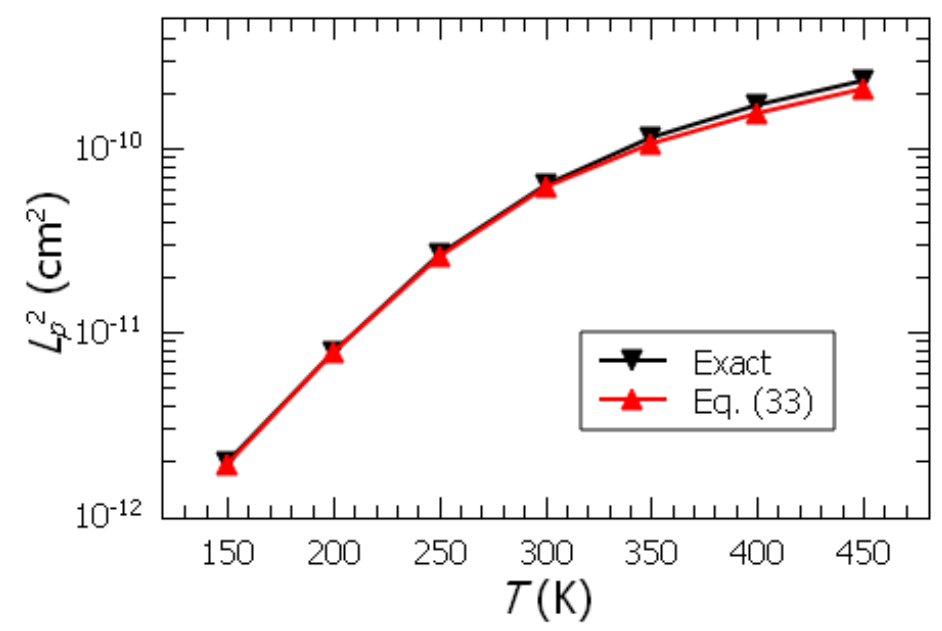

Figure 5. Diffusion length of holes squared vs temperature obtained with a typical generation rate of $G_{0}=10^{20} \mathrm{~cm}^{-3} \mathrm{~s}^{-1}$. The down triangles are the exact values, while the up triangles were obtained with equation (33).

The following is an evaluation of the complete procedure presented in subsection 2.4, 2.5, and 2.6 for the extraction of $L_{p}^{2}, \gamma_{p}$, and $\varepsilon c_{n}^{r} /\left(q \mu_{n}\right)$. Using the numerical simulation, we have calculated $\beta$ for seven values of $\Lambda$ between 1.1 and $9.0 \mu \mathrm{m}$ (similar to those achieved experimentally) and the six values of $\xi_{\text {ext }}$ used experimentally $\left(500 \leq \xi_{\text {ext }} \leq 5000 \mathrm{~V} \mathrm{~cm}^{-1}\right)$, for $G_{0}$ $=1 \times 10^{20} \mathrm{~cm}^{-3} \mathrm{~s}^{-1}$ and $G_{0}=2 \times 10^{20} \mathrm{~cm}^{-3} \mathrm{~s}^{-1}$ and seven different temperatures $(150 \mathrm{~K} \leq T \leq 450 \mathrm{~K})$. We have also calculated $\sigma_{0}$ to estimate $L_{n}^{2}$ from equation (51) and $\gamma_{n}$ from equation (18) for the same temperatures and generation rates. For each $G_{0}, T$ and $k$ value, we obtain $\varphi_{k}^{-2}$ and $L_{k}^{2}$ by fitting $(1-\beta)^{-1}$ vs $\left[q \xi_{\text {ext }} /\left(k_{b} T\right)\right]^{2}$ with equation (38). For each $G_{0}$ and $T$ value, we obtain $\varphi_{0}$ and $L_{D}^{2}$ by fitting $\varphi_{k}^{-1}$ vs $k^{2}$ with equation (40). Subsequently, $L_{S}^{2}$ and $L_{\xi}^{4}$ are obtained by fitting $L_{k}^{2}$ vs $k^{2}$ with equation (43) or equation (44). Finally, $L_{p}^{2}$ is obtained by replacing the values of $\gamma_{n}$, $L_{D}^{2}$, and $L_{\xi}^{4}$ in equation (33), and $\gamma_{p}$ is obtained from equation (27). These last two parameters: $L_{p}^{2}$ and $\gamma_{p}$, are obtained simultaneously by iteration until convergence, as was explained in subsection 2.5 .

The relative errors obtained for $L_{p}^{2}$ with $G_{0}=2 \times 10^{20} \mathrm{~cm}^{-3} \mathrm{~s}^{-1}$ are slightly lower than with $G_{0}$ $=10^{20} \mathrm{~cm}^{-3} \mathrm{~s}^{-1}$. Figure 6 presents the relative errors obtained for $L_{p}^{2}$ with the lower generation rate. It is observed that almost the same results are obtained when we fit $L_{k}^{2}$ vs $k^{2}$ with equations (43) or with (44), except for the highest temperature, where the more complex equation (43) produces a slightly better estimation. Note that for this simulated material, the relative errors obtained for $L_{p}^{2}$ with the new approach are more than ten times lower than with the usual approach based on the RZW formula, where the relative errors could be as large as 200 $\%$. 


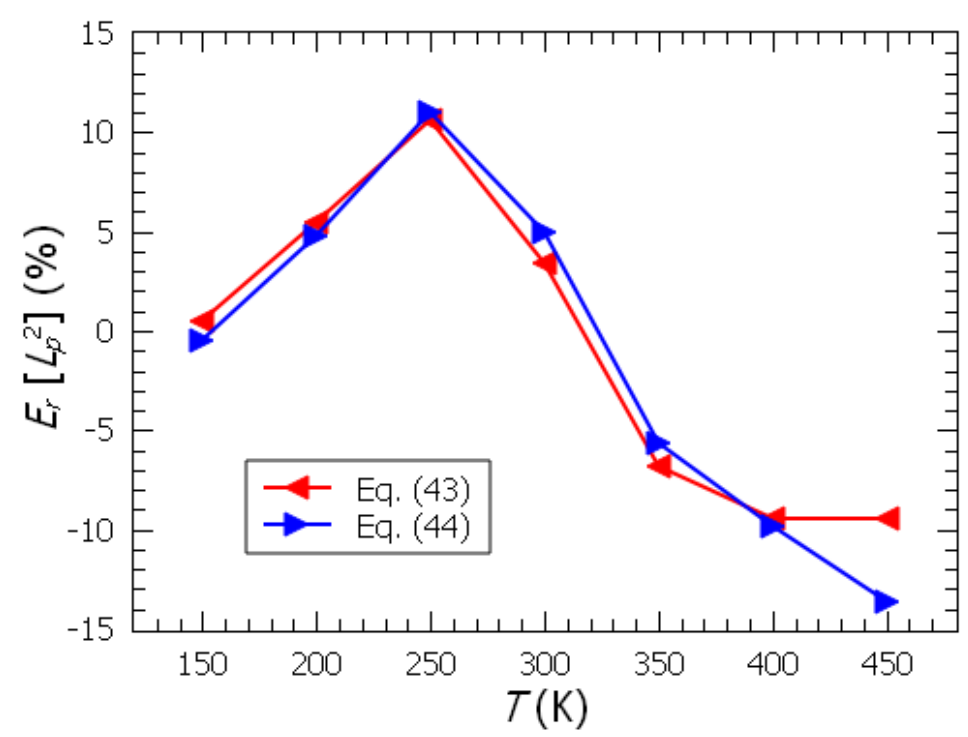

Figure 6. $L_{p}^{2}$ relative errors vs temperature obtained with the numerical simulation for $G_{0}=10^{20} \mathrm{~cm}^{-3} \mathrm{~s}^{-1}$ when using the procedure presented in subsection 2.5 and 2.6 for the estimation of $L_{p}^{2}$. In order to obtain $\gamma_{n}$ and $\gamma_{p}$, we have calculated the values of $\sigma_{0}$ and $\beta$ for two generation rates, $1 \times 10^{20}$ and $2 \times 10^{20} \mathrm{~cm}^{-3} \mathrm{~s}^{-1}$. The left and right triangles were obtained when using equations (43) and (44) for the fitting of $L_{k}^{2}$ vs $k^{2}$, respectively.

Figure 7 presents the estimates obtained for $\gamma_{p}$ and the exact values (circles). The diamonds were obtained from the values of $L_{D}^{2}$, using the approximate equation (45), while the triangles were obtained using the exact equation (27). As expected, we usually obtain a worst estimate with equation (45) than with equation (27), especially at low temperatures. In the first case, the relative errors obtained are lower than $11 \%$, while they are lower than $7 \%$ in the second one. $L_{D}^{2}$ severely overestimates $L_{a m b}^{2}$ at low temperatures, although the values of $\gamma_{p}$ found with the exact equation (27) and the approximate equation (45) are much more similar (compare figures 2 and 7). The left and right triangles were obtained using equations (43) and (44) for the fitting of $L_{k}^{2}$ vs $k^{2}$, respectively. Practically the same values of $\gamma_{p}$ are obtained with both equations. 


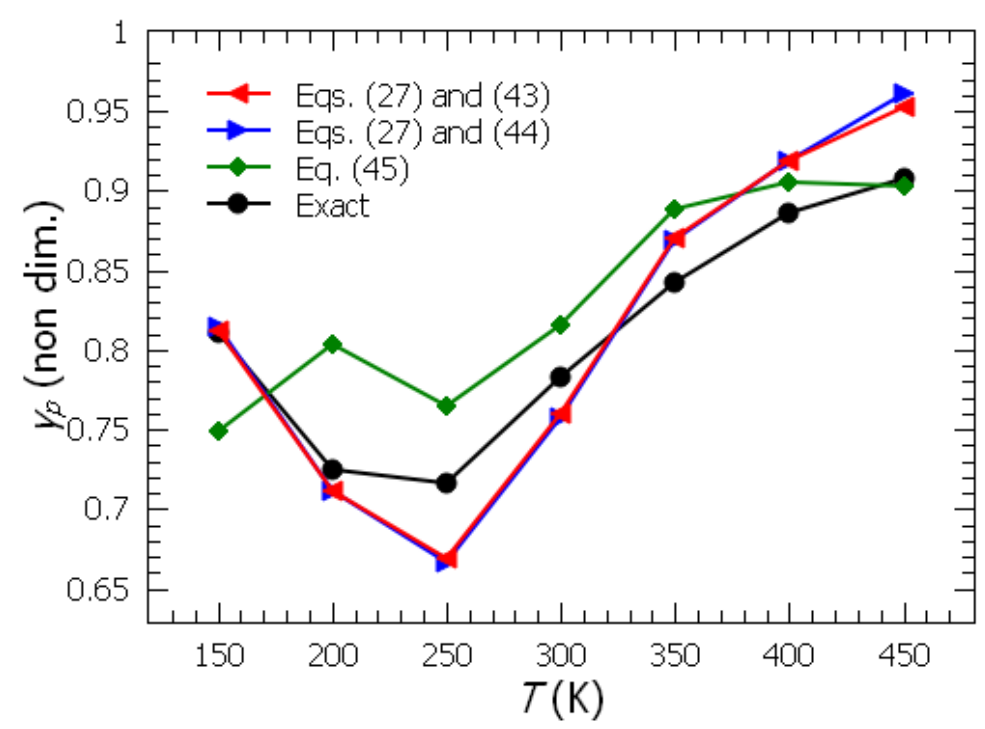

Figure 7. $\gamma_{p}$ values vs temperature obtained with the numerical simulation using a generation rate of $1.5 \times 10^{20} \mathrm{~cm}^{-3} \mathrm{~s}^{-1}$. The triangles were obtained using equation (27) and following the procedure presented in subsections 2.5 and 2.6 for two generation rate values, $1 \times 10^{20}$ and $2 \times 10^{20} \mathrm{~cm}^{-3} \mathrm{~s}^{-1}$. The diamonds were obtained from the values of $L_{D}^{2}$ using the approximate equation (45) and the circles correspond to the exact values.

In order to estimate $\tau_{d} / \tau_{p}^{t}$ or $\varepsilon c_{n}^{r} /\left(q \mu_{n}\right)$ for each generation rate and temperature, we replace the previously obtained values of $\gamma_{n}, L_{n}^{2}, \gamma_{p}$, and $L_{p}^{2}$ into equation (50). The points obtained with the higher generation rate present slightly higher errors and are displayed in figure 8 , where we also present the exact values for comparison. The results are practically independent of the fitting of $L_{k}^{2}$ vs $k^{2}$ with equations (43) or (44), except for the highest temperature. An excellent estimation of $\varepsilon c_{n}^{r} /\left(q \mu_{n}\right)$ is only obtained for temperatures equal to or lower than room temperature. For higher temperatures, we observe an overestimation of the exact value that increases with temperature. We obtain an overestimation of 56 or $69 \%$ for the highest temperature when $L_{k}^{2}$ vs $k^{2}$ is fitted with equations (43) or (44), respectively. The increase of the $\varepsilon c_{n}^{r} /\left(q \mu_{n}\right)$ overestimation with temperature is mainly due to the decrease in the ratio $L_{n}^{2} / L_{p}^{2}$ or $n_{0} / p_{0}$ with temperature (see figure 3 ), which produces underestimation in the values of $L_{p}^{2}$. For high temperatures, equation (40) produces a slight overestimation of $L_{D}^{2}$ (similarly to the RZW equation), which also contributes to the overestimation of $\varepsilon c_{n}^{r} /\left(q \mu_{n}\right)$. 


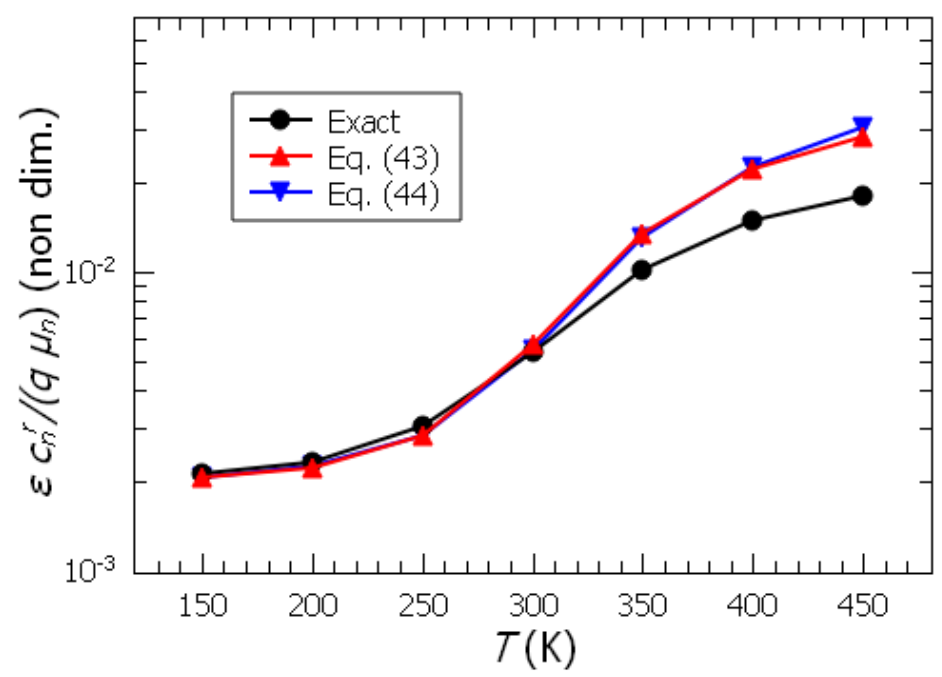

Figure 8. The triangles correspond to estimates of $\tau_{d} / \tau_{p}^{t}$ obtained with the procedure described in subsections $2.5,2.6$ and 2.7 while the circles correspond to the exact value. The increase in the errors of $\tau_{d} / \tau_{p}^{t}$ with temperature results from the decrease of the ratio $L_{n}^{2} / L_{p}^{2}$ with temperature (see figure 3).

In a a-Si:H-like material where $\varepsilon \sim 1.1 \times 10^{-12} \mathrm{~F} \mathrm{~cm}^{-1}$, we can estimate $c_{n}^{V B T} / \mu_{n}$ from the low-temperature values of $\varepsilon c_{n}^{r} /\left(q \mu_{n}\right)$, and $c_{n}^{D S} / \mu_{n}$ from its high-temperature values. $c_{n}^{V B T}$ and $c_{n}^{D S}$ are the electron capture coefficients in the region of the valence band tail and the deep states, respectively.

\section{Experimental}

\subsection{Procedure and sample details}

We performed SSPC and SSPG measurements on a a-Si:H and a pm-Si:H samples. The semiconductors were deposited on glass at $175{ }^{\circ} \mathrm{C}$ by Plasma Enhanced Chemical Vapor Deposition (PECVD) in a conventional capacitively-coupled RF-powered reactor [31]. The a-Si:H sample consists of $0.95 \mu \mathrm{m}$ of a-Si:H deposited at a rate of $0.81 \AA^{-1} \mathrm{~s}^{-1}$ from pure $\mathrm{SiH}_{4}$ at a flow rate of $30 \mathrm{sccm}$ with a RF power of $11 \mathrm{~mW} \mathrm{~cm}^{-2}$ and a chamber pressure of 0.067 Torr. The pm-Si:H sample consists of a very thin layer of a-Si:H $(\sim 14 \mathrm{~nm})$ deposited on the glass to ensure adhesion and $0.86 \mu \mathrm{m}$ of pm-Si:H deposited at a rate of $0.95 \AA^{s^{-1}}$ from a mixture of $\mathrm{SiH}_{4}$ (at a flow rate of 6 sccm) and $\mathrm{H}_{2}$ (at a flow rate of $500 \mathrm{sccm}$ ) with a $\mathrm{RF}$ power of $44 \mathrm{~mW} \mathrm{~cm}^{-2}$ and a chamber pressure of 2.2 Torr. A detailed study of the structure and hydrogen content of similar samples can be found in Ref. [32]. After the semiconductor deposition, we deposit two parallel silverpaste contacts separated by $1 \mathrm{~mm}$ on top of each sample.

The experimental setup is presented in figure 1. In order to gain precision, we chopped the weak beam at $20 \mathrm{~Hz}$ and measured the AC signals with a lock-in amplifier to obtain $\beta$. A linearly polarized He-Ne laser (633 nm) was used as a light source because its photon energy $(1.96 \mathrm{eV})$ is slightly higher than the mobility-gap energy of the semiconductors $(\sim 1.8 \mathrm{eV})$. The weak beam 
intensity was fixed at a value at least 50 times lower than that of the main beam. To minimize light-induced changes in the material during the experiments due to the Staebler-Wronski effect [19], the samples were light-soaked before the measurements for 2 hrs at room temperature using the maximum flux of the laser $\left(3 \times 10^{16} \mathrm{~cm}^{-2} \mathrm{~s}^{-1}\right)$.

For six different temperatures between 253 and $353 \mathrm{~K}$ and two light intensities, we measured $\beta$ for different values of $\Lambda$ between 1.1 and $9.0 \mu \mathrm{m}$ and six voltages between 50 and $500 \mathrm{~V}$. We estimate that the low and high photon fluxes $\left(1.5 \times 10^{16}\right.$ and $\left.3 \times 10^{16} \mathrm{~cm}^{-2} \mathrm{~s}^{-1}\right)$, produce in the semiconductors an average photocarrier generation rates of $5 \times 10^{19}$ and $1 \times 10^{20} \mathrm{~cm}^{-3} \mathrm{~s}^{-1}$, respectively.

The steady-state photoconductivity was also measured for the same light intensities, temperatures, and voltages, to evaluate the Ohmicity of the contacts. The equations presented in this manuscript assume contact Ohmicity, which is sometimes difficult to achieve experimentally, especially with slightly non-intrinsic materials [19]. Figure 9 presents the SSPC normalized to the value measured at $50 \mathrm{~V}$, versus the applied voltage (symbols). The points were obtained with the maximum flux at the extreme temperatures (253 and $353 \mathrm{~K}$ ) for each sample. The solid line corresponds to the ideal Ohmic behavior (linear behavior). The contacts inject extra-carriers for the pm-Si:H sample (superlinear behavior), while the contacts block the transport of carriers for the a-Si:H sample (sublinear behavior). In absolute value, the deviations from the ideal Ohmicity are practically the same for both samples at the lowest temperature (see figure 9). As the temperature increases, the pm-Si:H sample gradually becomes more Ohmic, while the a-Si:H sample progressively becomes less Ohmic. This difference can be appreciated in figure 9 by comparing the photocurrents of the samples at $353 \mathrm{~K}$. The same tendencies are observed for the low photon flux. To estimate $L_{n}^{2}$, we use the values of the current measured at $50 \mathrm{~V}$, where the influence of the contacts is the lowest.

In the following subsections, we present and discuss the experimental SSPG results and their implications. From now on, the (a) figures correspond to the a-Si:H sample, while the (b) figures correspond to the pm-Si:H sample. 


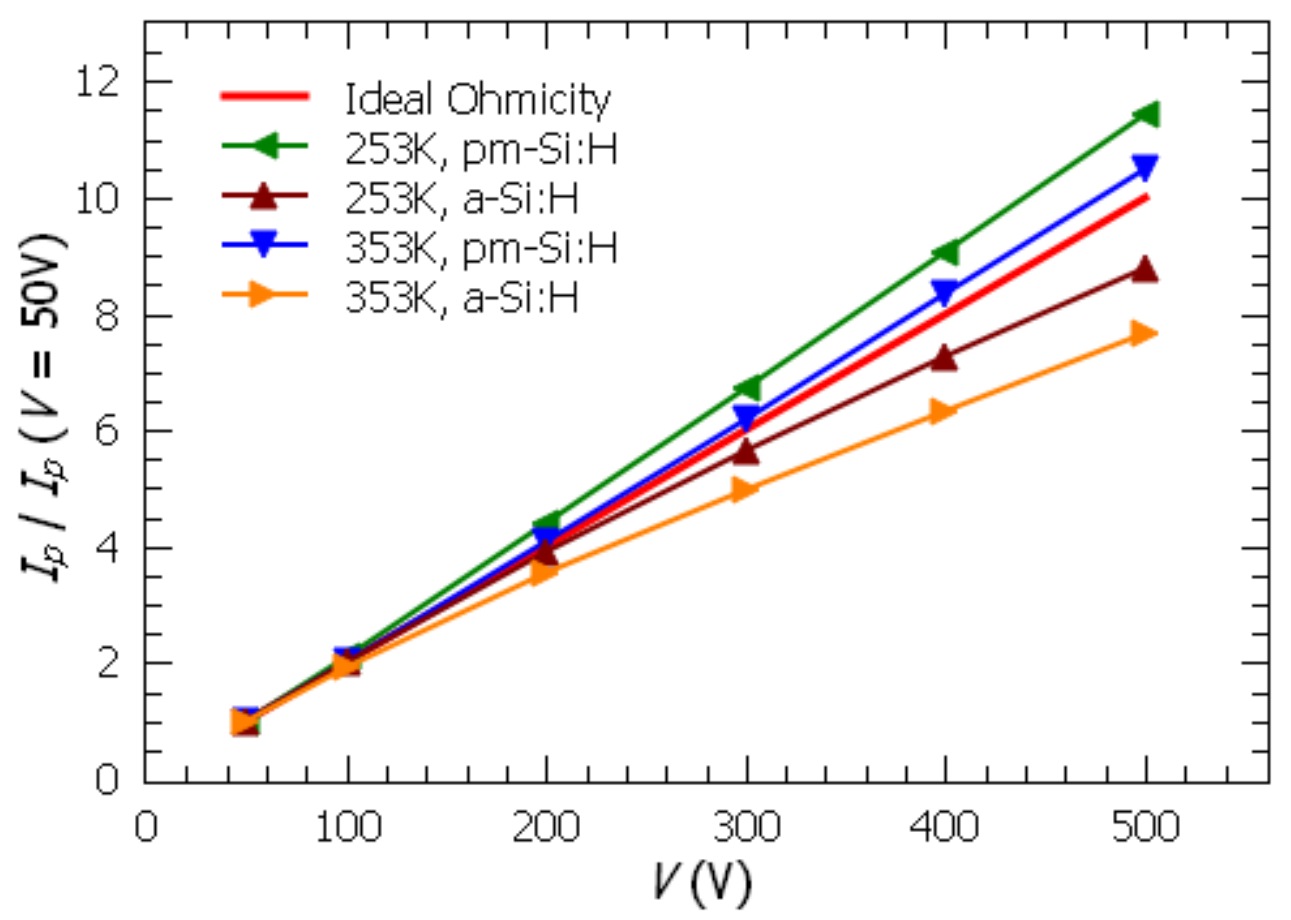

Figure 9. Steady-state photocurrent normalized to the value measured at $50 \mathrm{~V}$ vs the applied voltage. We observe that none of the two samples is perfectly ohmic, although the non-Ohmicities in the pm-Si:H are lower than in a-Si:H, especially at high temperatures.

\subsection{Extraction of $L_{D}^{2}, L_{\xi}^{4}$, and $\gamma_{\sigma}$}

Figure 10 presents the experimental plots of $(1-\beta)^{-1}$ versus $\left[q \xi_{\text {ext }} /\left(k_{b} T\right)\right]^{2}$ obtained with the high flux at $293 \mathrm{~K}$. For different $k$ values, we use different symbols. The solid lines correspond to the linear fittings of the points obtained for the same $k$ value using equation (38). In accordance with the theory, we observe an increase of the lines' intercepts with the ordinate axis $\left(\varphi_{k}^{-2}\right)$ and slopes $\left(L_{k}^{2}\right)$ as $k$ increases from 0.71 to $4.6 \mu \mathrm{m}^{-1}$. Similar results were obtained for the lower photon flux and the other temperatures with both photon fluxes (see figures S1-S6).
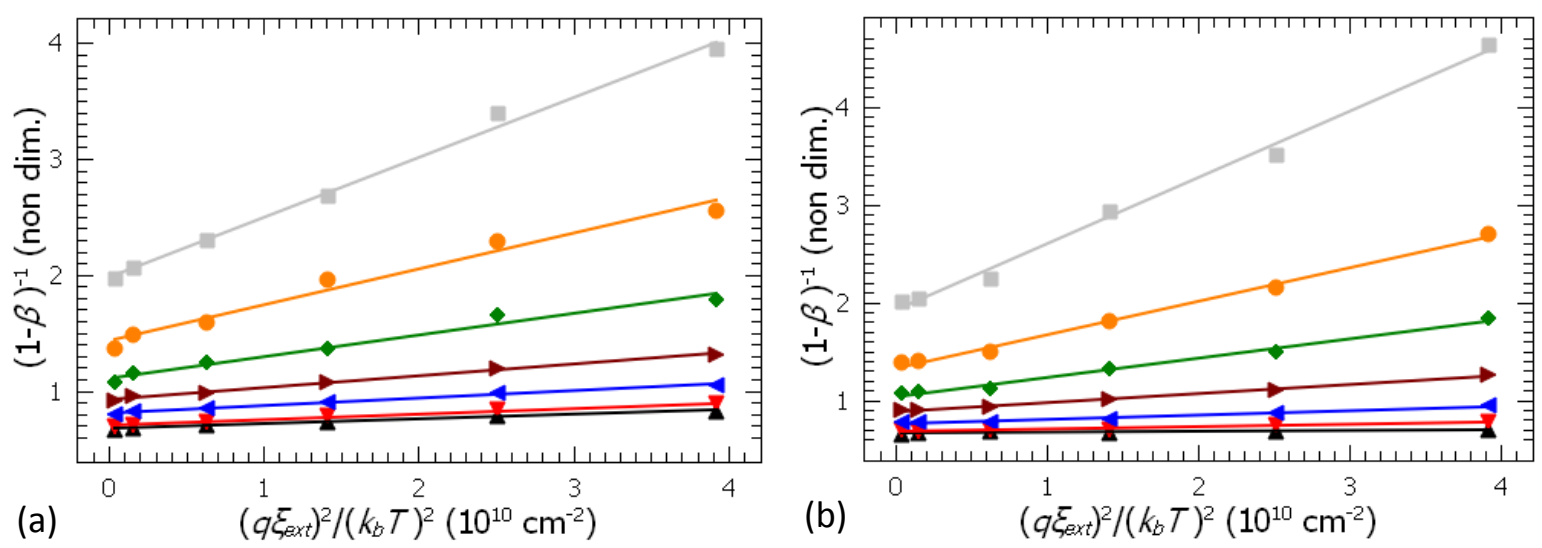
Figure 10. Each group of symbols corresponds to the experimental values of $(1-\beta)^{-1}$ versus $\left[q \xi_{\text {ext }} /\left(k_{b} T\right)\right]^{2}$ for a fix $k$ value, obtained at $293 \mathrm{~K}$ with the high light intensity for (a) the a-Si:H or (b) the pm-Si:H sample. The solid lines correspond to a linear fit of the experimental points with equation (38). An increase of the lines' intercepts $\left(\varphi_{k}^{-2}\right)$ and slopes $\left(L_{k}^{2}\right)$ with $k$ is observed, in agreement with the MT model.

Figures 11 and 12 present the previous values of $\varphi_{k}^{-1}$ vs $k^{2}$ and $L_{k}^{2}$ vs $k^{2}$, respectively; they were obtained from the fitting of the experimental data presented in figure 10. As predicted by the model, we observe a linear behavior for $\varphi_{k}^{-1}$ vs $k^{2}$ and a parabolic behavior for $L_{k}^{2}$ vs $k^{2}$. The solid lines in figure 11 correspond to the linear fit of $\varphi_{k}^{-1}$ vs $k^{2}$ with equation (40) to obtain $\varphi_{0}$ and $L_{D}^{2}$. The solid lines in figure 12 correspond to the fit of $L_{k}^{2}$ vs $k^{2}$ with equation (44) to obtain $L_{S}^{2}$ and $L_{\xi}^{4}$. We have also obtained good fittings for the low photon flux and the other temperatures with both photon fluxes (see figures S7-S18). We have omitted to present the fitting with the more complex equation (43), because practically the same values of $L_{\xi}^{4}$ were obtained in this way.
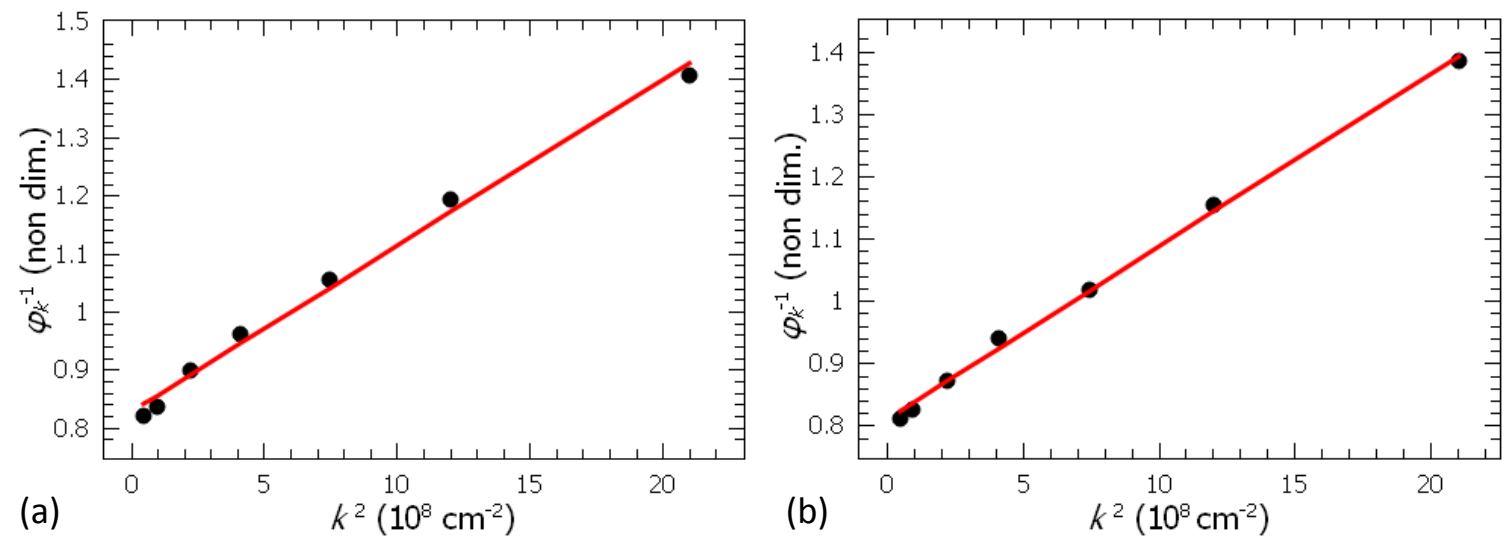

Figure 11. The symbols correspond to the experimental values of $\varphi_{k}^{-1}$ versus $k^{2}$ for (a) the a$\mathrm{Si}: \mathrm{H}$ and (b) the pm-Si:H sample, obtained with the maximum light intensity at $293 \mathrm{~K}$ from the fitting of the experimental data with equation (38). $\varphi_{0}$ and $L_{D}^{2}$ are obtained from the linear fitting of the symbols with equation (40) (solid lines).

A difference between both samples is observed with respect to the $L_{S}^{2}$ value (i.e., the intercept of the parabola with the ordinate axis). For the pm-Si:H sample, we obtain excellent fits of the experimental data without the necessity of including the extra parameter, $L_{S}^{2} \neq 0$ [see figure 12(b)]. On the contrary, a positive value of $L_{S}^{2}$ is usually needed for obtaining good fittings of the experimental data for the a-Si:H sample [see figure 12(a)]. Further research is needed in order to clarify the origin of this parameter. Although we have noted that it is possibly related to slightly blocking contacts (see figure 9), we do not discard the possible influence of surface recombination or non-homogeneities in the material, neither of which were considered in our model. 

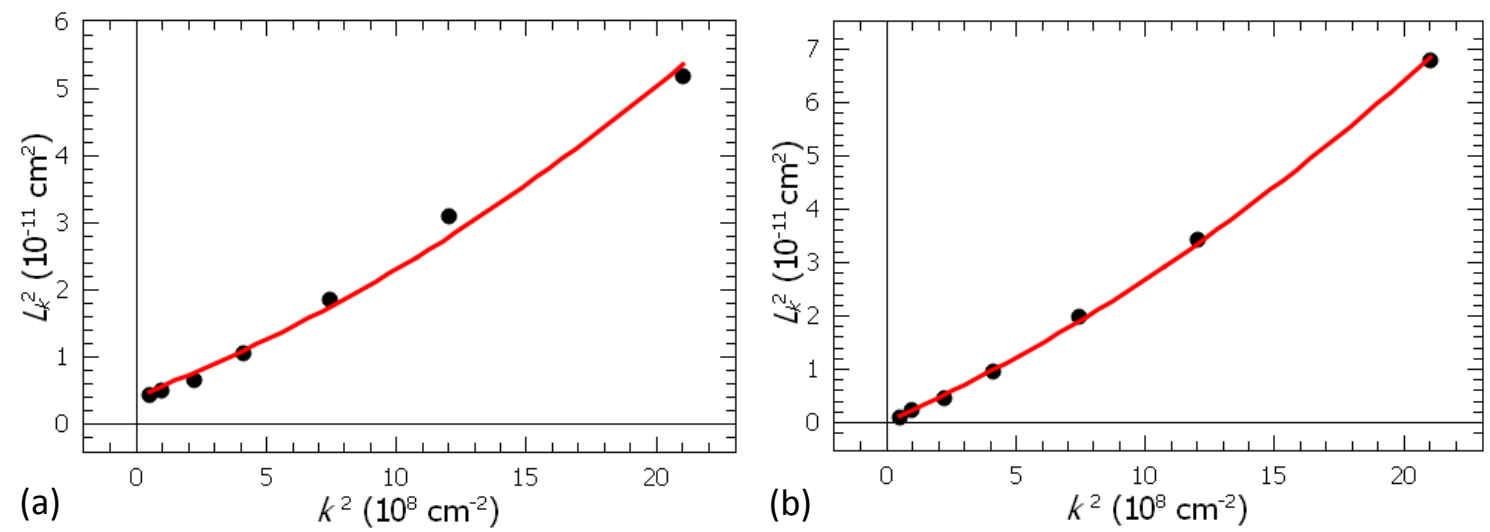

Figure 12. The symbols correspond to the experimental values of $L_{k}^{2}$ versus $k^{2}$ for (a) the a-Si:H and (b) the pm-Si:H sample, obtained with the maximum photon flux at $293 \mathrm{~K}$ from the fitting of the experimental data with equation (38). $L_{S}^{2}$ and $L_{\xi}^{4}$ are obtained from the fitting of the symbols with equation (44) (solid lines).

Figures 13 present the experimental values of $\gamma_{\sigma}$ for different temperatures. The circles in figure 13(a) were obtained from equation (18) by measuring SSPC for two nearby light intensities. The triangles correspond to the values of $\gamma_{\sigma}$ found from equation (34) assuming $\gamma_{0}^{2} /\left(1+G_{2} / G_{1}\right)=0.85$ and using the values of $\varphi_{0}$ obtained from the linear fit of $\varphi_{k}^{-1}$ vs $k^{2}$. In accordance with the numerical simulation, we find a decrease of $\gamma_{\sigma}$ for high temperatures and a slight variation of its value with the generation rate.

We have chosen the value of $\gamma_{0}^{2} /\left(1+G_{2} / G_{1}\right)=0.85$, because it produces the best agreement with the values obtained from SSPC in figure 13(a). Despite the good agreement, we observe that the values of $\gamma_{\sigma}$ found from SSPC are noisy. Therefore, we will use the values of $\gamma_{\sigma}$ calculated from $\varphi_{0}$ in the rest of this paper. We use the same value of $\gamma_{0}^{2} /\left(1+G_{2} / G_{1}\right)$ for both samples because this parameter only depends on the equipment calibration.
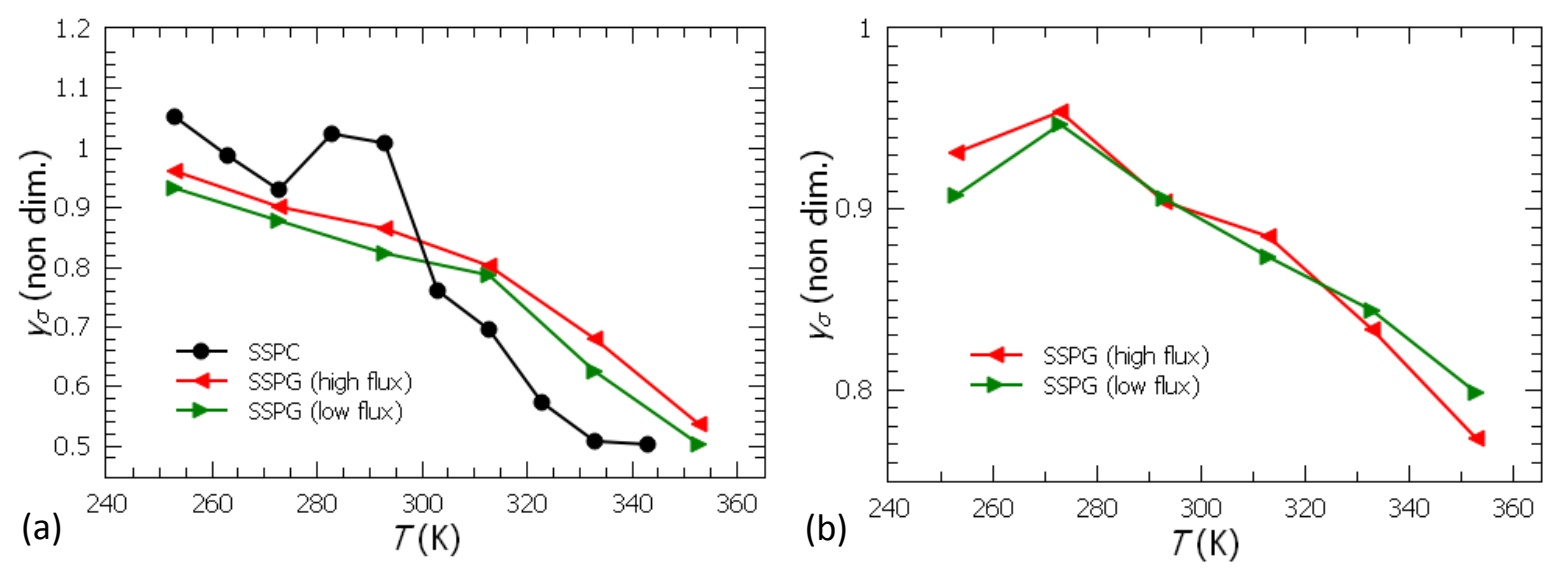

Figure 13. The symbols correspond to the experimental values of $\gamma_{\sigma}$ versus temperature, obtained for (a) the a-Si:H sample and (b) the pm-Si:H sample. The triangles were calculated from equation (34) assuming $\gamma_{0}^{2} /\left(1+G_{2} / G_{1}\right)=0.85$ and using the $\varphi_{0}$ values obtained from the 
linear fitting of $\varphi_{k}^{-1}$ versus $k^{2}$ for each photon flux. The circles in (a) were obtained by measuring SSPC for two nearby light intensities.

\subsection{Estimation of $L_{p}^{2}, \gamma_{p}, \varepsilon c_{n} /\left(q \mu_{n}\right)$ and the DOS}

Figures 14 present the values of $L_{p}^{2}$ obtained with the high light intensity for different temperatures, using the basic low-voltage approach (down triangles) and the multi-voltage approach (up triangles). Similar behaviors are observed with the low light intensity.
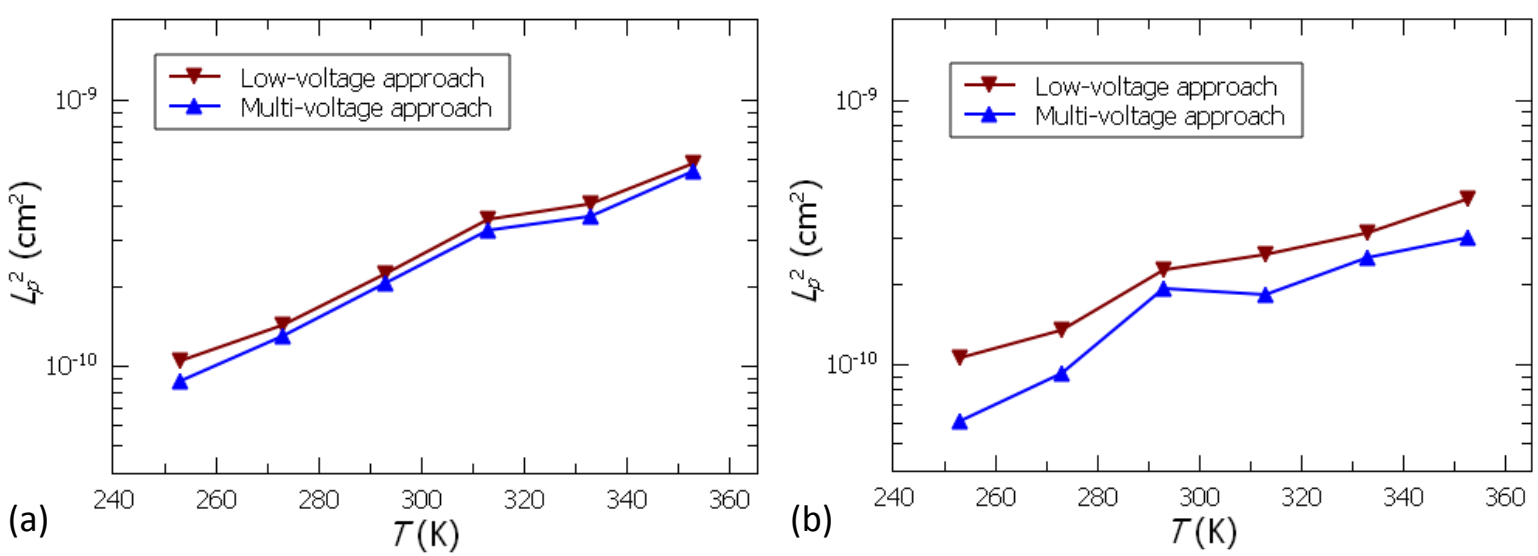

Figure 14. The symbols correspond to the hole diffusion length squared versus temperature, obtained with the high photon flux for (a) the a-Si:H sample and (b) the pm-Si:H sample. In accordance with the numerical simulation, it is observed that the usual low-voltage approach overestimates the values of $L_{p}^{2}$ found with the multi-voltage approach, especially at low temperatures.

As expected from our calculations, we observed that the low-voltage approach overestimates the value of $L_{p}^{2}$ obtained with the multi-voltage procedure, especially for the pmSi:H sample. The maximum overestimations are found at the lowest temperature for both samples in agreement with the numerical simulation. In this case, the low-voltage approach produces a $75 \%$ overestimation of $L_{p}^{2}$ with respect to the multi-voltage approach in the pm-Si:H sample, and just a $20 \%$ for the a-Si:H sample. For the pm-Si:H sample, excepting the points measured at $293 \mathrm{~K}$, we also find a steady increase of the relative overestimation as the temperature decreases, as was observed in the numerical simulation (see figure 2). The discrepancy obtained at $293 \mathrm{~K}$ is probably linked to the Staebler-Wronski effect [19]. Note that the measurement at $293 \mathrm{~K}$ was the first one we performed on the pm-Si:H sample, and the $L_{p}^{2}$ value obtained for this temperature is unexpectedly high. The light soaking of $2 \mathrm{hrs}$ before measurements was clearly not enough to stabilize the transport properties in the pm-Si:H sample.

By comparing the values of $L_{p}^{2}$ obtained with the multi-voltage approach in figures 14 , we observe that the a-Si:H sample presents larger values of $L_{p}^{2}$ than the pm-Si:H sample for all the temperatures. On average, $L_{p}^{2}$ in the a-Si:H sample is $\sim 60 \%$ larger than in the pm-Si:H sample. 
Note that the low-voltage approach could wrongly make us assume that the diffusion lengths at the two lowest temperatures are the same for both samples. Similarly, we have also observed that $L_{n}^{2}$ is larger in the a-Si:H sample for both light intensities at all the temperatures. On average, $L_{n}^{2}$ in the a-Si:H sample is $\sim 40 \%$ larger than in the pm-Si:H sample.

In the basic low-voltage SSPG [10], we first estimate $L_{D}^{2}$ and $\varphi_{0}$ for each photon flux from the fitting of the lowest voltage data $(50 \mathrm{~V})$ with the RZW equation. Then, we estimate $\gamma_{n}$ from $\varphi_{0}$ and $\gamma_{p}$ from $L_{D}^{2}$ using equations (34) and (45), respectively. Finally, $L_{p}^{2}$ is obtained for each light intensity from the formula $L_{p}^{2} \simeq L_{D}^{2} /\left(\gamma_{n}+\gamma_{p}\right)$. This one was the procedure used for extracting the points presented in figures 14. A simpler and more popular version of this approach is obtained by approximating $\gamma_{n}+\gamma_{p}$ by 2 in the previous formula to obtain $L_{p}^{2}$ directly from $L_{D}^{2}[6,11,16,29,30]$. With this extra approximation, the $L_{p}^{2}$ values are sometimes higher than those obtained from the multi-voltage approach.

Figures 15 presents the values of $\gamma_{p}$ obtained for the different temperatures using the two mentioned approaches. In both cases, and for all the temperatures, we obtained values of $\gamma_{p}<1$, as expected from the numerical simulation. In addition, the $\gamma_{p}$ values found from the multivoltage procedure are not very different from those found with the low-voltage approach (compare with figure 7). The increase of $\gamma_{p}$ with temperature obtained with the numerical simulation in the same temperature range is only observed for the a-Si:H sample. In the numerical simulation, the $\gamma_{p}$ values obtained from the low-voltage approach are usually larger than those obtained from the multi-voltage approach; this behavior is only seen for the pm-Si:H sample. The experimental errors affect more severely $\gamma_{p}$ than $L_{p}^{2}$, because $\gamma_{p}$ is obtained from a difference quotient.
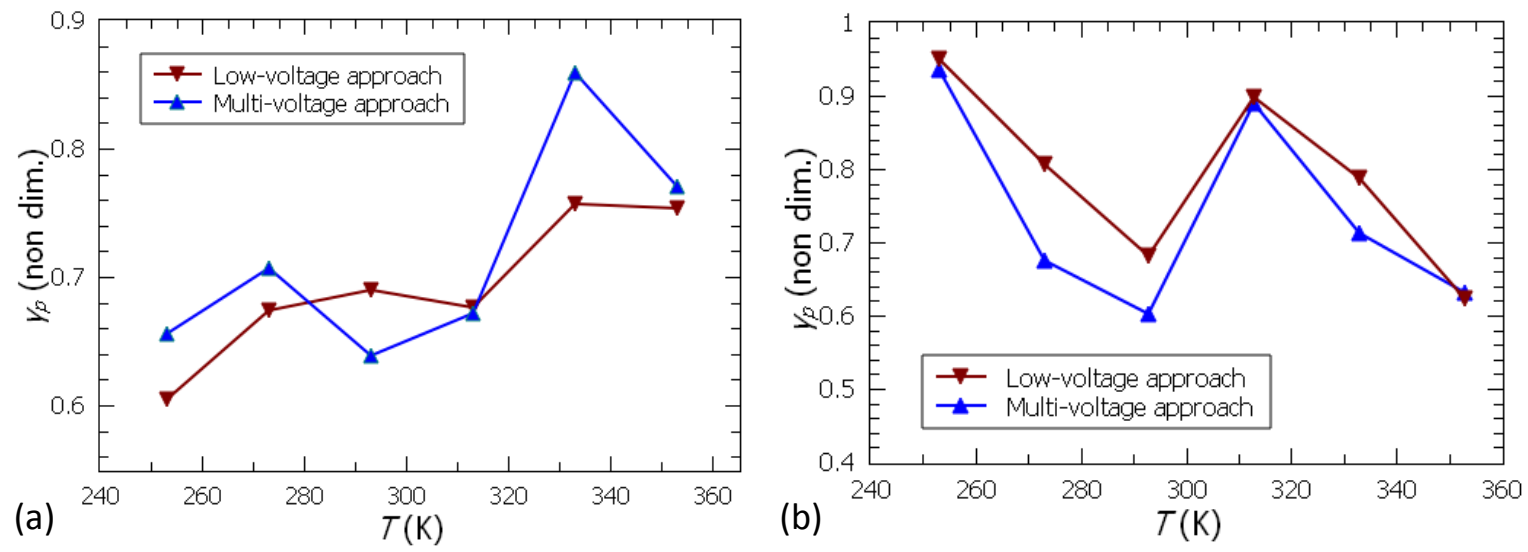

Figure 15. The symbols correspond to the experimental $\gamma_{p}$ values versus temperature, obtained for (a) the a-Si:H sample and (b) the pm-Si:H sample. The $\gamma_{p}$ values found with both approaches are lower than one and not very different from each other, as expected from the numerical simulation.

Figure 16 present the values of $\varepsilon c_{n}^{r} /\left(q \mu_{n}\right)$ versus temperature, obtained for each sample with both light intensities. The expected increase of $\varepsilon c_{n}^{r} /\left(q \mu_{n}\right)$ with temperature is just observed for the a-Si:H sample. However, it is much lower than in the numerical simulation, and 
it could be only due to the artifact produced by equation (50) at high temperatures (see figure 8). Therefore, the difference between the electron capture coefficients of the valence band tail and the deep states (of each sample) are much lower than in the numerical simulation. It is also known that the difference between these capture coefficients is responsible for producing values of $\gamma_{\sigma}$ greater than 1 (sensitization of the photoconductivity), as it is observed in the numerical simulation (see figure 4) [33]. For both samples we observed that the values of $\gamma_{\sigma}$ are lower than one (see figure 13), which is also consistent with a low difference between these capture coefficients. A slight difference between these two capture coefficients has already been reported for similar a-Si:H-like materials [34].

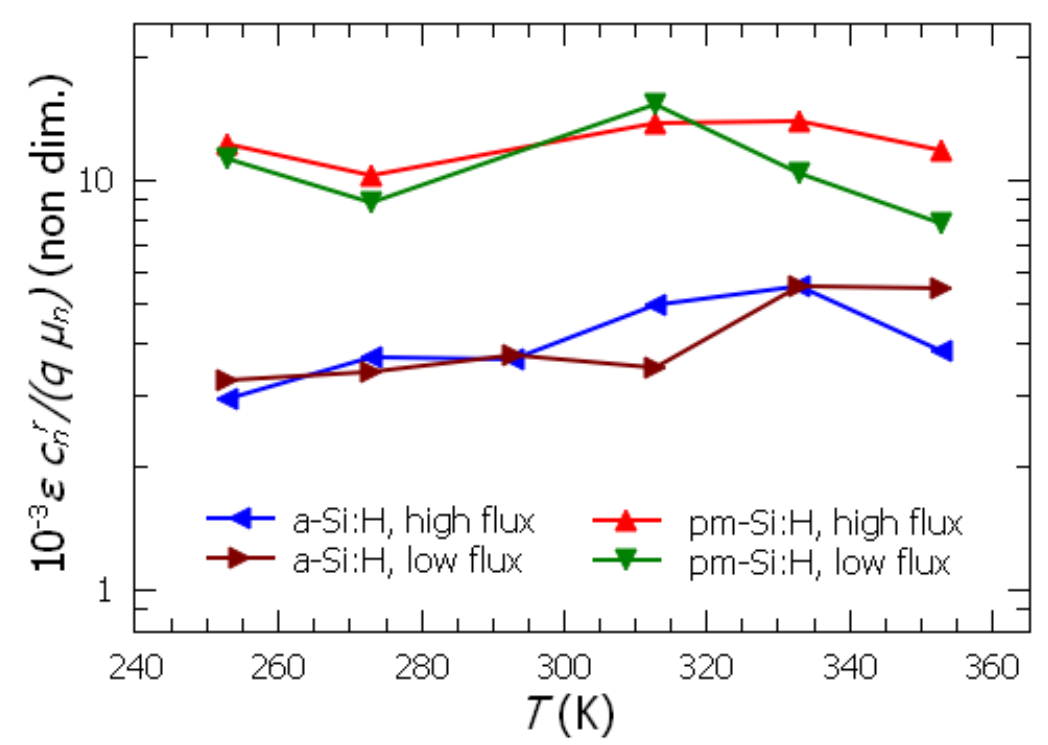

Figure 16. The symbols correspond to the values of $\varepsilon c_{n}^{r} /\left(q \mu_{n}\right)$ versus temperature, obtained from equation (50) for each sample with each photon flux. The $\varepsilon c_{n}^{r} /\left(q \mu_{n}\right)$ values are lower for the a-Si:H sample, which explains why $L_{n}^{2}$ is larger for this sample.

The relative overestimation of $L_{p}^{2}$ by the low-voltage approach increases with $L_{n}^{2} / L_{p}^{2}$ and $\varepsilon c_{n}^{r} /\left(q \mu_{n}\right)$ [see equation (52)]. The ratio $L_{n}^{2} / L_{p}^{2}$ does not change very much from one sample to the other, although $\varepsilon c_{n}^{r} /\left(q \mu_{n}\right)$ is more than two times higher for the pm-Si:H sample. As a consequence, it is the main responsible for the large discrepancies observed between the lowvoltage and multi-voltage approaches for this sample (see figures 14). The larger $L_{n}^{2}$ values observed for the a-Si:H sample are consistent with its lower $\varepsilon c_{n}^{r} /\left(q \mu_{n}\right)$ values.

Using the typical value $\varepsilon=1.1 \times 10^{-12} \mathrm{~F} \mathrm{~cm}^{-1}$ (corresponding to a-Si:H-like materials) and the results presented in figure 16, we can estimate the ratio $c_{n} / \mu_{n}$ for the valence band tail (VBT) and the deep states (DS) regions of the DOS. In the a-Si:H sample, we have $c_{n} / \mu_{n} \sim 5.1 \times$ $10^{-10} \mathrm{~V} \mathrm{~cm}$ for the VBT region and $c_{n} / \mu_{n} \sim 6.8 \times 10^{-10} \mathrm{~V} \mathrm{~cm}$ for the DS region. For the pm-Si:H sample, we have $c_{n} / \mu_{n} \sim 1.8 \times 10^{-9} \mathrm{~V} \mathrm{~cm}$ for the VBT and the DS regions.

Under the same assumptions, $\mu_{n} n_{0} \gg \mu_{p} p_{0}$ and $c_{n}(E) n_{0} \gg c_{p}(E) p_{0}$, it can be found $[6,23,24]$ a formula for estimating $N\left(E_{t n}\right)$ from the $\gamma_{\sigma}, \sigma_{0}$ and $c_{n}^{r} / \mu_{n}$ values, where $E_{t n} \simeq E_{C}+$ 
$k_{b} T \ln \left(\frac{\sigma_{0}}{q \mu_{n} k_{b} T N\left(E_{C}\right)}\right)$ is the electron quasi-Fermi energy. Figure 17 presents the DOS obtained for each sample by assuming the typical values $E_{C}=1.8 \mathrm{eV}$ and $q \mu_{n} N\left(E_{C}\right)=2 \times 10^{22} \mathrm{~V}^{-2} \mathrm{~s}^{-1} \mathrm{~cm}^{-1}$. We appreciate that the DOS in the DS region is higher for the a-Si:H sample. Therefore, the higher values of $L_{n}^{2}$ that we observe for the a-Si:H sample are not produced by a lower DOS, but mainly by a lower value of $\varepsilon c_{n}^{r} /\left(q \mu_{n}\right)$.

These results are in agreement with previous studies performed on sandwich configurations [35], which show that the DOS in the DS region is lower for the pm-Si:H samples than for the a-Si:H samples deposited at the same temperature.

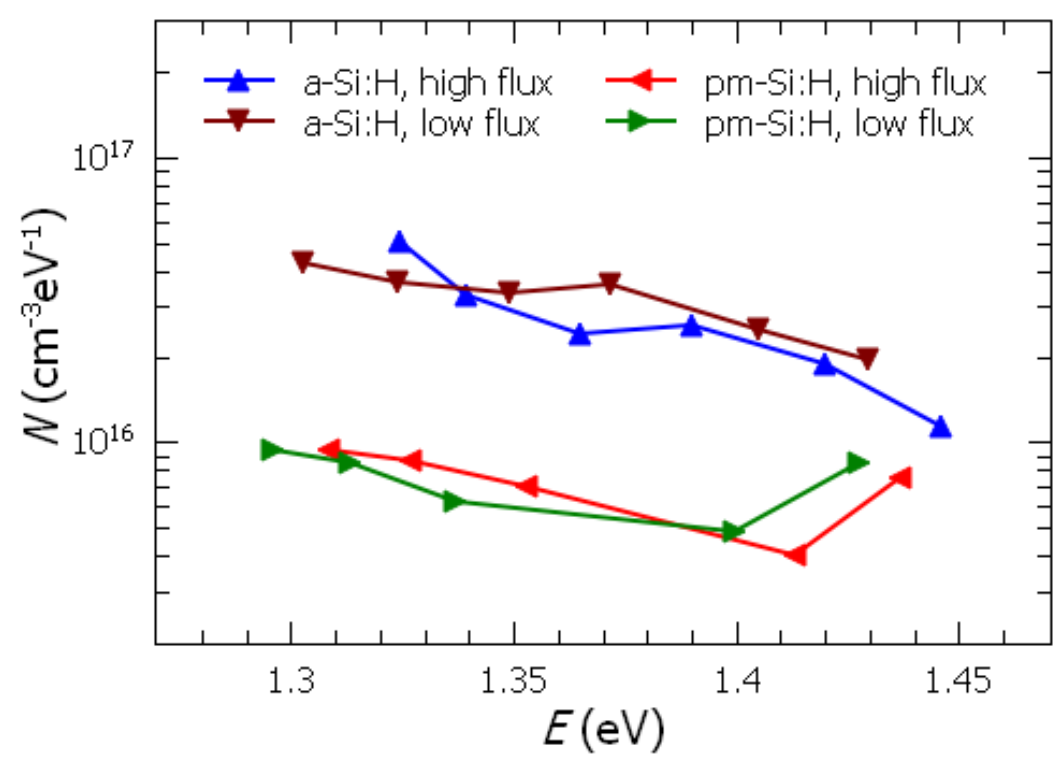

Figure 17. The symbols correspond to the DOS inside the band-gap obtained from the measurements of $\sigma_{0}, \gamma_{\sigma}$, and $c_{n}^{r} / \mu_{n}$. $\sigma_{0}$ was found from the SSPC whereas $\gamma_{\sigma}$ and $c_{n}^{r} / \mu_{n}$ were obtained using also the SSPG technique.

\section{Conclusion}

In this work, we have explored the limits of the low-voltage SSPG for the estimation of the minority-carrier diffusion length $\left(L_{p}\right)$. We have also presented a new alternative, the multivoltage SSPG, where $\beta$ is measured for light gratings with different periods under different applied voltages. The new method is much more precise, especially when the material presents high values of $L_{n}^{2} / L_{p}^{2}$ and $\varepsilon c_{n}^{r} /\left(q \mu_{n}\right)$ because the overestimation produced by the low-voltage approach is proportional to these values. Additionally, by combining the multi-voltage SSPG with the SSPC, $\varepsilon c_{n}^{r} /\left(q \mu_{n}\right)$ and the absolute DOS at the electron quasi-Fermi energy can be estimated.

After presenting the general expression of the current density for SSPG obtained from the MT model, we deduced the equations corresponding to the low-voltage and the multi-voltage SSPG. Then, we showed that the previous procedures for correcting the overestimations produced by the low-voltage approach $[2,4]$ do not work correctly. 
We have used a numerical simulation of an unintentionally-doped a-Si:H-like material to explore a wide range of temperatures $(150 \mathrm{~K} \leq T \leq 450 \mathrm{~K})$. The equations corresponding to the multi-voltage SSPG were obtained by assuming an extrinsic (n-type) material, where $\mu_{n} n_{0} \gg \mu_{p} p_{0}$ and $c_{n}(E) n_{0} \gg c_{p}(E) p_{0}$. The first inequality is verified in the simulated material, but the second is not strictly verified at high temperatures, which produces overestimations in the values of $\varepsilon c_{n}^{r} /\left(q \mu_{n}\right)$. The errors in the $L_{p}$ values obtained from the multi-voltage approach were consistently lower than 5\%. On the other hand, with the low-voltage approach, we have found $L_{p}$ overestimations of more than $100 \%$ for the lowest temperature, where the value of $L_{n}^{2} / L_{p}^{2}$ is maximum.

For the experimental evaluation, we have performed SSPC and SSPG experiments between 253 and $353 \mathrm{~K}$ on a a-Si:H and a pm-Si:H samples. We have also observed that the low-voltage approach produces overestimations of $L_{p}$ with respect to the values obtained with the multivoltage approach. Additionally, we noted that the overestimations decrease with temperature because of a decrease in the ratio $L_{n}^{2} / L_{p}^{2}$. We have always obtained larger overestimations for the pm-Si:H sample, because $\varepsilon c_{n}^{r} /\left(q \mu_{n}\right)$ was more than two times higher for this sample. For the a-Si:H sample at $253 \mathrm{~K}$, we obtained a $10 \%$ overestimation of $L_{p}$, while a $38 \%$ overestimation was observed for the pm-Si:H sample under the same conditions. We have found that the larger values of $L_{n}$ obtained for the a-Si:H sample (on average $20 \%$ larger than for the pm-Si:H sample) are not caused by a lower DOS but by a lower value of $c_{n}^{r} / \mu_{n}$.

Therefore, the multi-voltage SSPG is a very powerful technique to determine the minority carrier diffusion length with excellent precision. When combined with the SSPC experiment, characteristic quantities linked to fundamental transport parameters, such as the ratio between the recombination states' capture coefficient and mobility of majority carriers, can be extracted. Moreover, we obtain an absolute DOS spectroscopy above the Fermi energy.

\section{Acknowledgement}

We thank P. Roca I Cabarrocas from Laboratoire de Physique des Interfaces et des Couches Minces for providing the samples. We also thank J. Schmidt from Instituto de Física del Litoral for the discussion of the manuscript.

\section{Appendix A: Expressions of $Q^{+}, Q^{-}, \tau_{n}^{r}, \tau_{p}^{r}, \gamma_{n}$, and $\gamma_{p}$}

Under a steady-state uniform generation rate $G_{0}$, the total concentration of electrons is equal to the total concentration of holes,

$$
n^{t}+n=p^{t}+p
$$

and the recombination rate $r$, equals the generation rate, according to the steady-state continuity equation,

$$
r=G_{0}
$$


A slight variation in the steady-state uniform generation rate value $\delta G_{0}$, will induce small variations in the free electron and hole concentrations, $\delta n$ and $\delta p$, respectively. Differentiating equations (A1) and (A2) we obtain respectively,

$$
\begin{gathered}
\left(\frac{\partial n_{t}}{\partial n}\right)_{G_{0}} \delta n+\left(\frac{\partial n_{t}}{\partial p}\right)_{G_{0}} \delta p+\delta n=\left(\frac{\partial p_{t}}{\partial n}\right)_{G_{0}} \delta n+\left(\frac{\partial p_{t}}{\partial p}\right)_{G_{0}} \delta p+\delta p \\
\left(\frac{\partial r}{\partial n}\right)_{G_{0}} \delta n+\left(\frac{\partial r}{\partial p}\right)_{G_{0}} \delta p=\delta G_{0} .
\end{gathered}
$$

Using the definitions (7) and (8), we can rewrite the previous two equations respectively as:

$$
\begin{gathered}
\left(1+Q^{+}\right) \delta p=\left(1+Q^{-}\right) \delta n, \\
\frac{\delta p}{\tau_{p}^{r}}+\frac{\delta n}{\tau_{n}^{r}}=\delta G_{0} .
\end{gathered}
$$

Dividing equations (A5) and (A6) by $\delta G_{0}$, and taking the limit $\delta G_{0} \rightarrow 0$; we obtain equations (10) and (11), respectively. Combining equations (10) and (11), we can write the parameters $\gamma_{n}$ and $\gamma_{p}$ as a function of $Q^{+}, Q^{-}, \tau_{p}^{r}$ and $\tau_{n}^{r}$,

$$
\begin{aligned}
& \gamma_{n}=\frac{G_{0}\left(1+Q^{+}\right)}{n_{0}\left[\frac{\left(1+Q^{-}\right)}{\tau_{p}^{r}}+\frac{\left(1+Q^{+}\right)}{\tau_{n}^{r}}\right]}, \\
& \gamma_{p}=\frac{G_{0}\left(1+Q^{-}\right)}{p_{0}\left[\frac{\left(1+Q^{-}\right)}{\tau_{p}^{r}}+\frac{\left(1+Q^{+}\right)}{\tau_{n}^{r}}\right]} .
\end{aligned}
$$

In the following paragraphs, we deduce the expressions of $Q^{+}, Q^{-}, \tau_{p}^{r}$, and $\tau_{n}^{r}$ for a semiconductor with an arbitrary density of monovalent localized states within its mobility gap. The steady-state electron occupancy function of monovalent localized states with energy $E$ is given by,

$$
f_{n}(E)=\left[c_{n}(E) n+e_{p}(E)\right] \tau(E)
$$

where $\tau(E)=\left[c_{n}(E) n+c_{p}(E) p+e_{n}(E)+e_{p}(E)\right]^{-1}, c_{n}(E)\left[c_{p}(E)\right]$ is the electron (hole) capture coefficient of the localized states at energy $E$ and $e_{n}(E)\left[e_{p}(E)\right]$ is its electron (hole) emission coefficient, which are related by the detailed balance principle,

$$
e_{n}(E)=c_{n}(E) N_{C} \exp \left(\frac{E-E_{C}}{k_{b} T}\right), \quad e_{p}(E)=c_{p}(E) N_{V} \exp \left(\frac{E_{V}-E}{k_{b} T}\right)
$$

$E_{C}\left(E_{V}\right)$ separates the localized states from the free electron (hole) states. $N_{C}$ and $N_{V}$ are the effective density of states at the mobility edges and are usually approximated by $N_{C} \simeq$ $k_{b} T N\left(E_{C}\right)$ and $N_{V} \simeq k_{b} T N\left(E_{V}\right)$.

The steady-state total concentration of trapped charge is

$$
q\left(p_{t}-n_{t}\right)=q \int_{E_{V}}^{E_{C}}\left(1-f_{n}(E)\right) N^{D O N}(E) \mathrm{d} E-q \int_{E_{V}}^{E_{C}} f_{n}(E) N^{A C C}(E) \mathrm{d} E,
$$


where $N^{D O N}(E)\left(N^{A C C}(E)\right)$ is the density of donor-like (acceptor-like) localized states at the energy $E$. Replacing equation (A9) in equation (A11), and taking the derivatives given by equations (7) we obtain

$$
\begin{aligned}
& Q^{+}=\int_{E_{v}}^{E_{c}} c_{p}(E)\left(c_{n}(E) n_{0}+e_{p}(E)\right) \tau^{2}(E) N(E) \mathrm{d} E, \\
& Q^{-}=\int_{E_{v}}^{E_{c}} c_{n}(E)\left(c_{p}(E) p_{0}+e_{n}(E)\right) \tau^{2}(E) N(E) \mathrm{d} E,
\end{aligned}
$$

where $N(E)=N^{D O N}(E)+N^{A C C}(E)$.

The steady-state recombination rate is

$$
r=\left[n p-N_{C} N_{V} \exp \left(\frac{E_{V}-E_{C}}{k_{b} T}\right)\right] \int_{E_{V}}^{E_{C}} c_{n}(E) c_{p}(E) \tau(E) N(E) \mathrm{d} E .
$$

Replacing equation (A9) in (A14) and calculating the derivatives presented in equations (8) we obtain

$$
\begin{aligned}
& \frac{1}{\tau_{n}^{r}}=\int_{E_{v}}^{E_{c}} c_{n}(E)\left(c_{p}(E) p_{0}+e_{p}(E)\right)\left(c_{p}(E) p_{0}+e_{n}(E)\right) \tau^{2}(E) N(E) \mathrm{d} E \\
& \frac{1}{\tau_{p}^{r}}=\int_{E_{v}}^{E_{c}} c_{p}(E)\left(c_{n}(E) n_{0}+e_{n}(E)\right)\left(c_{n}(E) n_{0}+e_{p}(E)\right) \tau^{2}(E) N(E) \mathrm{d} E
\end{aligned}
$$

\section{Appendix B: Dependence of the drift mobilities with the carrier concentrations}

The electron and hole drift mobilities are defined respectively by $[2,4]$ :

$$
\mu_{N}=\frac{\mu_{n} n}{N}, \quad \mu_{P}=\frac{\mu_{n} p}{P},
$$

where $N$ and $P$ are respectively the total concentrations of electrons and holes (free plus trapped carriers). Under the MT model, $N$ and $P$ are a function of the free electron $(n)$ and hole $(p)$ concentrations. For simplicity, let us assume steady-state conditions and a monovalent density of localized states (see Appendix A),

$$
\begin{gathered}
N(n, p)=n+\int_{E_{V}}^{E_{C}} \frac{\left(c_{n} n+e_{p}(E)\right) N_{A C C}(E) \mathrm{d} E}{c_{n} n+c_{p} p+e_{n}(E)+e_{p}(E)} \\
P(n, p)=p+\int_{E_{V}}^{E_{C}} \frac{\left(c_{p} p+e_{n}(E)\right) N_{D O N}(E) \mathrm{d} E}{c_{n} n+c_{p} p+e_{n}(E)+e_{p}(E)} .
\end{gathered}
$$

Conversely, $n$ and $p$ are functions of $N$ and $P$. Therefore, $\partial \mu_{N} / \partial P$ and $\partial \mu_{P} / \partial N$ are respectively given by:

$$
\begin{aligned}
& \frac{\partial \mu_{N}}{\partial P}=\frac{\mu_{n}}{N} \times \frac{\partial n}{\partial P}=\frac{\frac{\mu_{n}}{N} \times \frac{\partial N}{\partial p}}{\frac{\partial N}{\partial p} \times \frac{\partial P}{\partial n}-\frac{\partial P}{\partial p} \times \frac{\partial N}{\partial n}}, \\
& \frac{\partial \mu_{P}}{\partial N}=\frac{\mu_{n}}{P} \times \frac{\partial p}{\partial N}=\frac{\frac{\mu_{n}}{P} \times \frac{\partial P}{\partial n}}{\frac{\partial P}{\partial n} \times \frac{\partial N}{\partial p}-\frac{\partial N}{\partial n} \times \frac{\partial P}{\partial p},}
\end{aligned}
$$


where the second equalities were obtained using the inverse function theorem. Replacing the derivatives of equations (B2) and (B3) in equations (B4) and (B5) it can be observed that the assumptions used in previous papers [2-4]: $\partial \mu_{N} / \partial P=0$ and $\partial \mu_{P} / \partial N=0$, are not valid.

\section{Appendix C: Approximation of $\tau_{p}^{r}$ and $\gamma_{p}$ for $c_{n}(E) n_{0} \gg c_{p}(E) p_{0}$}

The steady-state uniform recombination rate is equal to the uniform generation rate [36],

$$
G_{0}=\left[n_{0} p_{0}-N_{C} N_{V} \exp \left(\frac{E_{V}-E_{C}}{k_{b} T}\right)\right] \int_{E_{V}}^{E_{C}} \frac{c_{n}(E) c_{p}(E) N(E) \mathrm{d} E}{c_{n}(E) n_{0}+c_{p}(E) p_{0}+e_{n}(E)+e_{p}(E)} .
$$

As a consequence of the negative exponent, the second term inside the square bracket is much smaller than the first one and can be discarded. Discarding $c_{p} p_{0}$ in front of $c_{n} n_{0}$, multiplying and dividing the integrant by its denominator, a useful formula is obtained,

$$
G_{0}=n_{0} p_{0} \int_{E_{V}}^{E_{C}} \frac{c_{n}(E) c_{p}(E)\left[c_{n}(E) n_{0}+e_{n}(E)+e_{p}(E)\right] N(E) \mathrm{d} E}{\left[c_{n}(E) n_{0}+e_{n}(E)+e_{p}(E)\right]^{2}} .
$$

Discarding $c_{p} p_{0}$ in front of $c_{n} n_{0}$ in equation (A16), it is obtained

$$
\frac{1}{\tau_{p}^{r}}=\int_{E_{v}}^{E_{c}} \frac{c_{p}(E)\left[c_{n}(E) n_{0}+e_{n}(E)\right]\left[c_{n}(E) n_{0}+e_{p}(E)\right] N(E) \mathrm{d} E}{\left[c_{n}(E) n_{0}+e_{n}(E)+e_{p}(E)\right]^{2}} .
$$

Distributing the numerator and using equations (A10),

$$
\frac{1}{\tau_{p}^{r}}=\int_{E_{v}}^{E_{c}} \frac{c_{p}(E) c_{n}(E)\left\{c_{n}(E) n_{0}^{2}+n_{0}\left[e_{n}(E)+e_{p}(E)\right]+c_{p}(E) N_{C} N_{V} \exp \left(\frac{E_{V}-E_{C}}{k_{b} T}\right)\right\} N(E) \mathrm{d} E}{\left[c_{n}(E) n_{0}+e_{n}(E)+e_{p}(E)\right]^{2}} .
$$

Discarding the third term in front of the first one, inside the numerator square bracket, due to its negative exponent, and using equation (C2), we obtain:

$$
\frac{1}{\tau_{p}^{r}} \simeq \frac{G_{0}}{p_{0}}=\frac{1}{\tau_{p}}
$$

Replacing (C5) in equation (A8) we obtain

$$
\gamma_{p}=\frac{1}{1+\frac{\left(1+Q^{+}\right) \tau_{p}}{\left(1+Q^{-}\right) \tau_{n}^{r}}}<1
$$

It can be observed that $\gamma_{p}<1$ because the second term inside the denominator is positive.

\section{References}

[1] Ritter D, Zeldov E and Weiser K 1986 Steady-state photocarrier grating technique for diffusion length measurement in photoconductive insulators Appl. Phys. Lett. 49 791-3

[2] Ritter D, Zeldov E and Weiser K 1988 Ambipolar transport in amorphous semiconductors in the lifetime and relaxation-time regimes investigated by the steady-state photocarrier grating technique Phys. Rev. B 38 8296-304 
[3] Li Y-M 1990 Phototransport under the presence of a small steady-state photocarrier grating Phys. Rev. B 42 9025-32

[4] Hattori K, Okamoto H and Hamakawa Y 1992 Theory of the steady-state-photocarriergrating technique for obtaining accurate diffusion-length measurements in amorphous silicon Phys. Rev. B 45 1126-38

[5] Abel C D, Bauer G H and Bloss W H 1995 Generalized theory for analytical simulation of the steady-state photocarrier grating technique Philos. Mag. B Phys. Condens. Matter; Stat. Mech. Electron. Opt. Magn. Prop. 72 551-62

[6] Schmidt J A and Longeaud C 2005 Analysis of the steady-state photocarrier grating method for the determination of the density of states in semiconductors Phys. Rev. B $\mathbf{7 1}$ 125208

[7] Merazga A, Tobbeche S, Main C, Al-Shahrani A and Reynolds S 2006 Numerical simulation of the steady state photoconductivity in hydrogenated amorphous silicon including localized state electron hopping J. Phys. Condens. Matter 18 3721-34

[8] Longeaud C and Tobbeche S 2009 The influence of hopping on modulated photoconductivity J. Phys. Condens. Matter 21045508

[9] Balberg I 1990 The theory of the photoconductance under the presence of a small photocarrier grating J. Appl. Phys. 67 6329-33

[10] Shah A, Sauvain E, Hubin J, Pipoz P and Hof C 1997 Free carrier ambipola Philos. Mag. B Phys. Condens. Matter; Stat. Mech. Electron. Opt. Magn. Prop. 75 925-36

[11] Brüggemann R 2016 Steady-State Photocarrier Grating Method Advanced Characterization Techniques for Thin Film Solar Cells (Weinheim, Germany: Wiley-VCH) pp 163-88

[12] Ritter D, Weiser K and Zeldov E 1987 Steady-state photocarrier grating technique for diffusion-length measurement in semiconductors: Theory and experimental results for amorphous silicon and semi-insulating GaAs J. Appl. Phys. 62 4563-70

[13] Duboz J Y, Binet F, Dolfi D, Laurent N, Scholz F, Off J, Sohmer A, Briot O and Gil B 1997 Diffusion length of photoexcited carriers in GaN Mater. Sci. Eng. B 50 289-95

[14] Adhyaksa G W P, Veldhuizen L W, Kuang Y, Brittman S, Schropp R E I and Garnett E C 2016 Carrier Diffusion Lengths in Hybrid Perovskites: Processing, Composition, Aging, and Surface Passivation Effects Chem. Mater. 28 5259-63

[15] Veldhuizen L W, Adhyaksa G W P, Theelen M, Garnett E C and Schropp R E I 2017 Benchmarking photoactive thin-film materials using a laser-induced steady-state photocarrier grating Prog. Photovoltaics Res. Appl. 25 605-13

[16] Longeaud C, Ramos F J, Rebai A and Rousset J 2018 Impact of Environmental Stresses Onto Transport Properties of Hybrid Perovskite Investigated by Steady State Photocarrier Grating and Steady State Photocurrent Techniques Sol. RRL 21800192

[17] Singh J and Shimakawa K 2003 Advances in amorphous semiconductors (London: Taylor \& Francis)

[18] Powell M J and Deane S C 1993 Improved defect-pool model for charged defects in amorphous silicon Phys. Rev. B 48 10815-27 
[19] Street R A 1991 Hydrogenated amorphous silicon (Cambridge: Cambridge University Press)

[20] Stepanov S 2001 Photo-electromotive-force effect in semiconductors Handbook of Advanced Electronic and Photonic Materials and Devices (San Diego, USA: Academic Press) pp 205-72

[21] Moore A R 1984 Chapter 7 Diffusion Length in Undoped a-Si: H Semicond. Semimetals 21 239-56

[22] Shah A, Hubin J, Sauvin E, Pipoz P, Beck N and Wyrsch N 1993 Role of dangling bond charge in determining $\mu \tau$ products for a-Si:H J. Non. Cryst. Solids 164-166 485-8

[23] Longeaud C, Schmidt J A and Kleider J P 2006 Determination of semiconductor band gap state parameters from photoconductivity measurements. I. Theoretical developments Phys. Rev. B - Condens. Matter Mater. Phys. 73235316

[24] Longeaud C, Schmidt J A and Koropecki R R 2006 Determination of semiconductor band gap state parameters from photoconductivity measurements. II. Experimental results Phys. Rev. B - Condens. Matter Mater. Phys. 73235317

[25] Halpern $\mathrm{V} 1986$ The statistics of recombination via dangling bonds in amorphous silicon Philos. Mag. B 54 473-82

[26] Simmons J G and Taylor G W 1972 Theory of steady state photoconductivity in amorphous semiconductors J. Non. Cryst. Solids 8-10 947-53

[27] Tran M Q 1995 On thermal quenching of the photoconductivity in hydrogenated amorphous silicon Philos. Mag. B 72 35-66

[28] Willemen J A 1998 Modelling of Amorphous Silicon Single- and Multi-Junction Solar Cells (Doctoral Thesis, Delft: TU Delft)

[29] Kopprio L, Longeaud C and Schmidt J A 2019 Hydrogenated amorphous silicon characterization from steady state photoconductive measurements Semicond. Sci. Technol. 34045010

[30] Kopprio L, Longeaud C and Schmidt J 2017 Obtainment of the density of states in the band tails of hydrogenated amorphous silicon J. Appl. Phys. 122085702

[31] Roca I Cabarrocas P, Kim K H, Cariou R, Labrune M, Johnson E V., Moreno M, Torres Rios A, Abolmasov S and Kasouit S 2012 Low temperature plasma synthesis of nanocrystals and their application to the growth of crystalline silicon and germanium thin films Mater. Res. Soc. Symp. Proc. 1426 319-29

[32] Morral A F i, Cabarrocas P R i and Clerc C 2004 Structure and hydrogen content of polymorphous silicon thin films studied by spectroscopic ellipsometry and nuclear measurements Phys. Rev. B 69125307

[33] Taylor G W and Simmons J G 1976 Superlinearity and sensitization in defect photoconductors J. Phys. C Solid State Phys. 9 1013-23

[34] Schmidt J A, Longeaud C, Koropecki R R and Kleider J P 2006 Determination of the density of states of semiconductors from steady-state photoconductivity measurements $J$. Non. Cryst. Solids 352 1024-7

[35] Kleider J P, Longeaud C, Gauthier M, Meaudre M, Meaudre R, Butté R, Vignoli S and Roca I 
Cabarrocas P 1999 Very low densities of localized states at the Fermi level in hydrogenated polymorphous silicon from capacitance and space-charge-limited current measurements Appl. Phys. Lett. 75 3351-3

[36] Simmons J G and Taylor G W 1971 Nonequilibrium Steady-State Statistics and Associated Effects for Insulators and Semiconductors Containing an Arbitrary Distribution of Traps Phys. Rev. B 4 502-11 